QUARTERLY OF APPLIED MATHEMATICS

VOLUME LXVIII, NUMBER 2

JUNE 2010, PAGES 349-374

S 0033-569X(10)01185-3

Article electronically published on February 19, 2010

\title{
FREE ENERGIES FOR INCOMPRESSIBLE VISCOELASTIC FLUIDS
}

\author{
BY \\ G. AMENDOLA \\ Dipartimento di Matematica Applicata "U. Dini", via F. Buonarroti 1c, 56127-Pisa, Italy \\ In memory of Tristano Manacorda, Member of the Lincei Academy, Emeritus Professor of the Faculty \\ of Engineering of the University of Pisa, Italy (Firenze, October 24, 1920 - Pisa, May 20, 2008)
}

\begin{abstract}
In this work we consider some expressions for the free energy, already proposed and studied for viscoelastic solids, and adapt them to incompressible viscoelastic fluids. The internal dissipation corresponding to each of these various forms of the free energy is also evaluated. In particular, the form of the minimum free energy for the discrete spectrum model is also considered in order to show its equivalence to some classical free energies.
\end{abstract}

1. Introduction. Many relevant studies have been done on the free energy of materials with memory. Such studies, both in the linear case (see, for example, [5]) and in terms of abstract formulations of thermodynamics [7, have shown that the free energy is not necessarily uniquely defined for these materials. Therefore, there may be many free energies associated with any given state of these bodies; moreover, they form a bounded and convex set with a minimum and a maximum element. Consequently, the determination of explicit forms for the free energy has become an interesting problem and several expressions have been proposed for viscoelastic solids (see, in alphabetical order, [5], 8 - 12, 15]-17, [19]-24 and 27]).

The aim of this article is to examine expressions for the free energy that have already been considered for viscoelastic solids, in order to adapt them to incompressible viscoelastic fluids.

This problem has been partially studied in [1]-[3], where expressions for the GraffiVolterra free energy, the most general quadratic form for the free energy [5] in the alternative form derived by Golden in 21 and the new formula proposed by Fabrizio in several of his works related to viscoelastic solids (see, for example, [10]) have been derived for our fluids. Also the minimum free energy, the importance of which is due to the fact

Received June 5, 2008 and, in revised form, March 12, 2009.

2000 Mathematics Subject Classification. Primary 74D05, 76A10, 30E20.

Key words and phrases. Viscoelastic fluid, fading memory, free energy.

This work was performed under the support of C.N.R. and M.I.U.R.

E-mail address: amendola@dma.unipi.it 
that it coincides with the maximum recoverable work obtainable from a given state of the body, has been derived in [1] and 3 in the frequency domain, following Golden's method used in 21] for viscoelastic solids.

In this work we recall the results related to these free energies and show that the classical free energies proposed by Day and Dill and the maximum free energy 17 can also be adapted to our fluids; the corresponding internal dissipations are derived too. Then, by using the results obtained for the minimum free energy corresponding to the case of relaxation functions given by the sum of exponentials [3], we are able to derive the kernel $\mu(s, u)$, introduced for the general form of free energy; this expression allows us to show an equivalence to the Breuer-Onat formula 4. We also give the form of the kernel $K(s, u)$, which yields the corresponding rate of dissipation. Finally, we show that the Day free energy is a special case of the minimum free energy (for the analogous results related to viscoelastic solids, see [21]).

The layout of this paper is as follows. In Section 2, the fundamental equations are given together with the consequences of the thermodynamic constraint. In Section 3, states and processes are defined as well as an equivalence relation between states introduced by means of the response of the material. In Section 4, the notion of work and the thermodynamic principles are considered, together with the defining properties of the free energy, of the maximum free energy and of the minimum one. In Section 5, some expressions for the work, corresponding to particular relative strain histories, are derived; moreover, an equivalent way of defining an equivalence relation between states is given by means of the work. In Section 6, various forms of the free energy are examined and the corresponding internal dissipations are evaluated. In Section 7, the minimum free energy for a discrete spectrum model is given in order to show the equivalence of this minimum free energy to Breuer-Onat's functional and, when the relaxation function is expressed by only one exponential, to Day's functional.

2. Basic relationships. We consider the linear theory of viscoelasticity for a viscoelastic fluid studied in [6], 26] and [31, for example. For such a fluid, assumed isotropic and incompressible, the linearization of the constitutive equation of Boltzmann type gives the following expression:

$$
\mathbf{T}(\mathbf{x}, t)=-p(\mathbf{x}, t) \mathbf{I}+2 \int_{0}^{+\infty} \mu^{\prime}(\mathbf{x}, s) \mathbf{E}_{r}^{t}(\mathbf{x}, s) d s .
$$

This relation yields the symmetric stress tensor $\mathbf{T}$ as a function of the infinitesimal strain $\mathbf{E}=\frac{1}{2}\left[\nabla \mathbf{u}+(\nabla \mathbf{u})^{T}\right], \mathbf{u}$ being the displacement vector, through its relative history

$$
\mathbf{E}_{r}^{t}(\mathbf{x}, s)=\mathbf{E}^{t}(\mathbf{x}, s)-\mathbf{E}(\mathbf{x}, t) \quad \forall s \in \mathbb{R}^{++} \equiv(0,+\infty),
$$

where $\mathbf{E}(\mathbf{x}, t) \in$ Sym denotes the current value of the strain, while $\mathbf{E}^{t}(\mathbf{x}, s)=\mathbf{E}(\mathbf{x}, t-s)$ $\forall s \in \mathbb{R}^{++}$is its past history. Moreover, the position vector, the dependence of which will be omitted later on, is $\mathbf{x} \in \Omega$, where $\Omega$ is a smooth bounded domain of $\mathbb{R}^{3}$, with a smooth boundary $\partial \Omega$, that is occupied by the fluid. Finally, $\mathbf{I}$ is the identity second-order tensor and $p$ is a scalar function which yields the reaction pressure due to the constraint of 
incompressibility and is constitutively indeterminate. The material function $\mu^{\prime}$ is related to the shear relaxation function,

$$
\mu(s)=-\int_{s}^{+\infty} \mu^{\prime}(\xi) d \xi \quad \forall s \in \mathbb{R}^{+} \equiv[0,+\infty),
$$

which is supposed to belong to $L^{1}\left(\mathbb{R}^{+}\right)$, to have the properties required by the thermodynamic laws and to be established by the following theorem [18].

Theorem 2.1. The constitutive equation (2.1) for linear viscoelastic fluids is compatible with the Second Law of Thermodynamics if and only if for every relaxation function $\mu \in L^{1}\left(\mathbb{R}^{+}\right)$such that $\int_{0}^{+\infty} \mu(s) d s \neq 0$, the following inequality holds:

$$
\int_{0}^{+\infty} \mu(s) \cos \omega s d s>0 \quad \forall \omega \in \mathbb{R} .
$$

Later on we shall be concerned with the frequency domain; thus, we recall the Fourier transform of any function $f: \mathbb{R} \rightarrow \mathbb{R}^{n}$, defined by

$$
f_{F}(\omega)=\int_{-\infty}^{+\infty} f(s) e^{-i \omega s} d s=f_{-}(\omega)+f_{+}(\omega) \quad \forall \omega \in \mathbb{R}
$$

where we have put

$$
f_{-}(\omega)=\int_{-\infty}^{0} f(s) e^{-i \omega s} d s, \quad f_{+}(\omega)=\int_{0}^{+\infty} f(s) e^{-i \omega s} d s
$$

also recall the half-range Fourier sine and cosine transforms

$$
f_{s}(\omega)=\int_{0}^{+\infty} f(s) \sin \omega s d s, \quad f_{c}(\omega)=\int_{0}^{+\infty} f(s) \cos \omega s d s .
$$

We note that if $f$ is a real-valued function, then we have $f_{F}^{*}(\omega)=f_{F}(-\omega)$, where * denotes the complex conjugate. Obviously, the definitions of $f_{+}, f_{s}$ and $f_{c}$ stand even if $f$ is defined on $\mathbb{R}^{+}$, as does the definition of $f_{-}$when $f$ is defined on $\mathbb{R}^{-} \equiv(-\infty, 0]$.

We can extend the domain of any $f$ defined on $\mathbb{R}^{+}$to $\mathbb{R}$ as follows. If we identify $f$ with a function defined on $\mathbb{R}$ that vanishes identically on the strictly negative reals $\mathbb{R}^{--} \equiv(-\infty, 0)$, we have

$$
f_{F}(\omega)=f_{+}(\omega)=f_{c}(\omega)-i f_{s}(\omega)
$$

the extensions of $f$ made by means of an odd function, i.e. $f(s)=-f(-s) \forall s<0$, or an even function, i.e. $f(s)=f(-s) \forall s<0$, yield

$$
f_{F}(\omega)=-2 i f_{s}(\omega), \quad f_{F}(\omega)=2 f_{c}(\omega),
$$

respectively.

We consider the following subsets of the complex $z$-plane $\mathbb{C}$ :

$$
\mathbb{C}^{-}=\left\{z \in \mathbb{C} ; \operatorname{Im} z \in \mathbb{R}^{-}\right\}, \quad \mathbb{C}^{(-)}=\left\{z \in \mathbb{C} ; \operatorname{Im} z \in \mathbb{R}^{--}\right\}
$$

and

$$
\mathbb{C}^{+}=\left\{z \in \mathbb{C} ; \operatorname{Im} z \in \mathbb{R}^{+}\right\}, \quad \mathbb{C}^{(+)}=\left\{z \in \mathbb{C} ; \operatorname{Im} z \in \mathbb{R}^{++}\right\},
$$

so that $\mathbb{C}^{ \pm}$include the real axis $\mathbb{R}$ while $\mathbb{C}^{( \pm)}$exclude it. 
The functions defined in (2.6) can be extended for any $z \in \mathbb{C}$; thus, we obtain two functions $f_{ \pm}(z)$ which are analytic in $\mathbb{C}^{(\mp)}$. They become analytic in $\mathbb{C}^{\mp}$, which include $\mathbb{R}$, by assuming their analyticity on the real axis $[21$. We shall indicate that the zeros and singularities of $f$ are only in $\mathbb{C}^{ \pm}$by means of the notation $f_{( \pm)}(z)$.

Using the Fourier transform (2.7) 2 , the thermodynamic restriction (2.4) becomes

$$
\mu_{c}(\omega)>0 \quad \forall \omega \in \mathbb{R}
$$

under the hypothesis of Theorem 2.1 given by

$$
\int_{0}^{+\infty} \mu(s) d s=\mu_{c}(0) \neq 0 .
$$

If we suppose that $\mu^{\prime} \in L^{1}\left(\mathbb{R}^{+}\right) \cap L^{2}\left(\mathbb{R}^{+}\right)$, then (2.8) gives

$$
\mu_{F}^{\prime}(\omega)=\mu_{c}^{\prime}(\omega)-i \mu_{s}^{\prime}(\omega) \quad \forall \omega \in \mathbb{R} .
$$

This Fourier transform belongs to $L^{2}(\mathbb{R})$ and, by virtue of the assumption of analyticity on $\mathbb{R}$ for the Fourier transforms, yields a function $\mu_{F}^{\prime}(z)$ that is analytic in $\mathbb{C}^{-}$. Moreover, we note that $\mu_{c}^{\prime}(\omega)$ is an even function of $\omega$, while $\mu_{s}^{\prime}(\omega)$, which is an odd function, vanishes at the origin and, by assumption, does so linearly.

We now recall some results derived in 19 and, in particular, in [1.

If $\mu^{\prime} \in L^{1}\left(\mathbb{R}^{+}\right)$and $\mu^{\prime \prime} \in L^{1}\left(\mathbb{R}^{+}\right)$, then we have

$$
\mu_{s}^{\prime}(\omega)=-\omega \mu_{c}(\omega), \quad \omega \mu_{s}^{\prime}(\omega)=\mu^{\prime}(0)+\mu_{c}^{\prime \prime}(\omega) ;
$$

moreover, from (2.15) 1 , taking account of the thermodynamic restriction (2.12), it follows that

$$
\omega \mu_{s}^{\prime}(\omega)<0 \quad \forall \omega \neq 0 .
$$

Using the inverse Fourier transform, we have

$$
\mu(s)=\frac{2}{\pi} \int_{0}^{+\infty} \mu_{c}(\omega) \cos \omega s d \omega,
$$

whence, by virtue of (2.15) 1 , we obtain

$$
\mu(0)=\frac{2}{\pi} \int_{0}^{+\infty} \mu_{c}(\omega) d \omega=-\frac{1}{\pi} \int_{-\infty}^{+\infty} \frac{\mu_{s}^{\prime}(\omega)}{\omega} d \omega>0 .
$$

Therefore, it follows that

$$
\mu(s)-\mu(0)=\frac{2}{\pi} \int_{0}^{+\infty} \mu_{c}(\omega)[\cos \omega s-1] d \omega<0, \quad \mu^{\prime}(0) \leq 0 .
$$

Furthermore, from (2.15) we get the asymptotic behaviours

$$
\lim _{\omega \rightarrow+\infty} \omega \mu_{s}^{\prime}(\omega)=-\lim _{\omega \rightarrow+\infty} \omega^{2} \mu_{c}(\omega)=\mu^{\prime}(0)<0
$$

under the hypothesis that $0 \neq\left|\mu^{\prime}(0)\right|<+\infty$. In particular, already in [1, we have derived the asymptotic behaviours of the Fourier transform $\mu_{F}^{\prime}(\omega)$, expressed by (2.14) in terms of $\mu_{c}^{\prime}(\omega)$ and $\mu_{s}^{\prime}(\omega)$ :

$$
\mu_{s}^{\prime}(\omega) \sim \frac{\mu^{\prime}(0)}{\omega}, \quad \mu_{c}^{\prime}(\omega) \sim-\frac{\mu^{\prime \prime}(0)}{\omega^{2}} .
$$


Finally, if we consider the even extension of $\mu$ to $\mathbb{R}$, then we have the odd extension for $\mu^{\prime}$ and, using (2.9) $)_{1}$ and $(2.15)_{1}$, we get

$$
\left[\mu_{F}^{\prime}(\omega)\right]^{*}=2 i \mu_{s}^{\prime}(\omega)=-2 i \omega \mu_{c}(\omega) .
$$

Therefore, by virtue of Plancherel's theorem and this last result, the constitutive equation (2.1) can be written in the frequency domain as

$$
\tilde{\mathbf{T}}(t)=\mathbf{T}(t)+p(t) \mathbf{I}=\frac{2 i}{\pi} \int_{-\infty}^{+\infty} \mu_{s}^{\prime}(\omega) \mathbf{E}_{r+}^{t}(\omega) d \omega=-\frac{2 i}{\pi} \int_{-\infty}^{+\infty} \omega \mu_{c}(\omega) \mathbf{E}_{r+}^{t}(\omega) d \omega
$$

Here, $\tilde{\mathbf{T}}$ denotes the extra stress, which is the stress due only to the relative strain history $\mathbf{E}_{r}^{t}(s)$, the Fourier transform of which is denoted by $\mathbf{E}_{r+}^{t}(\omega)$.

3. States and processes. The mechanical properties of any simple material are described by means of constitutive equations, which can be described in terms of states and processes (see, for example, [6] and [14]). Also the fluid, characterized by the linearized relation (2.1), can be considered as a simple material.

A mechanical process $P$ is a piecewise continuous map $P:[0, d) \rightarrow$ Lin, the set of second order tensors, defined by

$$
P(\tau)=\mathbf{L}^{P}(\tau) \quad \forall \tau \in[0, d),
$$

where the superscript $P$ denotes the values of the velocity gradient $\mathbf{L}=\nabla \mathbf{v}$ corresponding to the time interval $[0, d)$, where $d$ is the duration of the process.

Given a process $P \in \Pi$, the set of mechanical processes, its restriction to an interval $\left[\tau_{1}, \tau_{2}\right) \subset[0, d)$ is also a process, $P_{\left[\tau_{1}, \tau_{2}\right)} \in \Pi$. Moreover, it is useful to consider the composition of the processes $P_{j} \in \Pi$ with durations $d_{j}(j=1,2)$, defined by

$$
P_{1} * P_{2}(\tau)= \begin{cases}P_{1}(\tau) & \forall \tau \in\left[0, d_{1}\right), \\ P_{2}\left(\tau-d_{1}\right) & \forall \tau \in\left[d_{1}, d_{1}+d_{2}\right),\end{cases}
$$

which also belongs to $\Pi$.

The state transition function $\rho: \Sigma \times \Pi \rightarrow \Sigma$ maps any initial state $\sigma^{i} \in \Sigma$, the set of all possible states, and each process $P \in \Pi$ into the final state $\sigma^{f}=\rho\left(\sigma^{i}, P\right)$. Thus, if the initial state is $\sigma^{i}=\sigma(0)$, via a process $P_{[0, \tau)}$ we reach the final state $\sigma(\tau)=\rho\left(\sigma(0), P_{[0, \tau)}\right)$; if we have $\sigma(d)=\rho(\sigma(0), P)=\sigma(0)$, then the pair $(\sigma, P)$ is said to be a cycle.

For our model the state $\sigma$ is given by the relative history up to time $t$ of the infinitesimal strain tensor $\mathbf{E}$, that is,

$$
\sigma(t)=\mathbf{E}_{r}^{t}(s) \quad \forall s \in \mathbb{R}^{++} .
$$

Moreover, since the constitutive equation (2.1) involves the strain, we observe that it is convenient to consider a definition of a process which is less general than (3.1), by considering the strain rate tensor $\mathbf{D}=\left(\mathbf{L}+\mathbf{L}^{T}\right) / 2$, that is, the symmetric part of $\mathbf{L}$. Henceforth, since by virtue of the linear approximation we have $\mathbf{D}=\dot{\mathbf{E}}$, we shall assume that

$$
P(\tau)=\dot{\mathbf{E}}_{P}(\tau) \quad \forall \tau \in[0, d) .
$$

We denote by $t \geq 0$ the instant when any process can be applied.

If $t>0$ is the initial instant when the state is $\sigma(t)=\mathbf{E}_{r}^{t}(s)$, then $P(\tau)=\dot{\mathbf{E}}_{P}(\tau) \equiv$ $\dot{\mathbf{E}}(t+\tau)$. The extra stress, defined in $(2.23)_{1}$, can be derived from (2.1) by considering 
the integral over $(0,+\infty)$ as the sum of two integrals, the first between 0 and $\tau$ and the second between $\tau$ and $+\infty$; integrating by parts in the first integral, we have

$$
\tilde{\mathbf{T}}(t+\tau)=\mathbf{T}(t+\tau)+p(t+\tau) \mathbf{I}=2 \int_{0}^{\tau} \mu(s) \dot{\mathbf{E}}^{t+\tau}(s) d s-\breve{\mathbf{I}}^{t}\left(\tau, \mathbf{E}_{r}^{t}\right),
$$

where

$$
\breve{\mathbf{I}}^{t}\left(\tau, \mathbf{E}_{r}^{t}\right)=-2 \int_{0}^{+\infty} \mu^{\prime}(\xi+\tau) \mathbf{E}_{r}^{t}(\xi) d \xi, \quad \tau \in \mathbb{R}^{+} .
$$

If $P$ is applied to the state $\sigma(0)=\mathbf{E}_{r}^{0}(s) \forall s \in \mathbb{R}^{++}$at time $t=0$, then $\tau \equiv t \in[0, d)$; thus, (3.5) becomes

$$
\tilde{\mathbf{T}}(t)=\mathbf{T}(t)+p(t) \mathbf{I}=2 \int_{0}^{t} \mu(\eta) \dot{\mathbf{E}}^{t}(\eta) d \eta-\breve{\mathbf{I}}^{0}\left(t, \mathbf{E}_{r}^{0}\right),
$$

where the expression for $\breve{\mathbf{I}}^{0}\left(t, \mathbf{E}_{r}^{0}\right)$ can be deduced from (3.6) by putting $\tau=t$ and $t=0$, that is,

$$
\breve{\mathbf{I}}^{0}\left(t, \mathbf{E}_{r}^{0}\right)=-2 \int_{0}^{+\infty} \mu^{\prime}(\xi+t) \mathbf{E}_{r}^{0}(\xi) d \xi
$$

We note that if we have a constant history $\mathbf{E}^{t}(s)=\mathbf{E}^{\dagger}(s)=\mathbf{E} \forall s \in \mathbb{R}^{+}$to which the relative strain history $\mathbf{E}_{r}^{t}(s)=\mathbf{0}_{r}^{\dagger}(s)=\mathbf{0} \forall s \in \mathbb{R}^{++}$corresponds, then the extra stress, defined by (2.23) 1 with (2.1), becomes

$$
\tilde{\mathbf{T}}(t)=\mathbf{0} .
$$

Furthermore, we observe that a static continuation of duration $\tau \in \mathbb{R}^{++}$of the relative strain history, defined as

$$
\mathbf{E}_{r_{c}}^{t+\tau}(s)= \begin{cases}\mathbf{0} & \forall s \in(0, \tau] \\ \mathbf{E}_{r}^{t}(s-\tau) & \forall s>\tau\end{cases}
$$

yields the following expression for the extra stress:

$$
\begin{aligned}
\tilde{\mathbf{T}}(t+\tau) & =\mathbf{T}(t+\tau)+p(t+\tau) \mathbf{I}=2 \int_{\tau}^{+\infty} \mu^{\prime}(s) \mathbf{E}_{r_{c}}^{t+\tau}(s) d s \\
& =2 \int_{0}^{+\infty} \mu^{\prime}(\xi+\tau) \mathbf{E}_{r}^{t}(\xi) d \xi .
\end{aligned}
$$

The negative of such an extra stress coincides with the quantity $\breve{\mathbf{I}}^{t}$ introduced in (3.6). We also have the asymptotic limit

$$
\lim _{\tau \rightarrow+\infty} \tilde{\mathbf{T}}(t+\tau)=\mathbf{0}
$$

Bearing in mind (3.11) 3 , we can introduce the space of relative strain histories as follows:

$$
\boldsymbol{\Gamma}_{r}=\left\{\mathbf{E}_{r}^{t}: \mathbb{R}^{++} \rightarrow \text { Sym; }\left|\int_{0}^{+\infty} \mu^{\prime}(\xi+\tau) \mathbf{E}_{r}^{t}(\xi) d \xi\right|<+\infty \quad \forall \tau \in \mathbb{R}^{+}\right\} .
$$

Finally, we observe that $\tilde{\mathbf{T}}$, given by (2.1), is a state function, that is, it depends on the state only. 
Definition 1. Two states $\sigma_{j}(t)=\mathbf{E}_{r_{j}}^{t}(j=1,2)$ are said to be equivalent if the subsequent states $\sigma_{j}(t+\tau)=\mathbf{E}_{r_{j}}^{t+\tau}(j=1,2)$ satisfy the identity

$$
\tilde{\mathbf{T}}\left(\sigma_{1}(t+\tau)\right)=\tilde{\mathbf{T}}\left(\sigma_{2}(t+\tau)\right) \quad \forall \tau \in[0, d)
$$

for all $P \in \Pi$ of duration $d$.

In [2] we proved the following theorem.

Theorem 3.1. For a viscoelastic fluid of type (2.1), two states $\sigma_{j}(t)=\mathbf{E}_{r_{j}}^{t}(j=1,2)$ are equivalent if and only if

$$
\int_{0}^{+\infty} \mu^{\prime}(\xi+\tau)\left[\mathbf{E}_{r_{1}}^{t}(\xi)-\mathbf{E}_{r_{2}}^{t}(\xi)\right] d \xi=\mathbf{0} \quad \forall \tau \in \mathbb{R}^{++}
$$

Definition 1 gives a characterization of the states which are indistinguishable because they yield the same extra stress. Thus, we can consider an equivalence relation $\mathcal{R}$ in $\Sigma$ and its quotient space $\Sigma_{\mathcal{R}}$, the elements of which are the equivalence classes, denoted by $\sigma_{\mathcal{R}}$ and called minimal states of the fluid. Consequently, (3.15) is an equivalent form of (3.14) and, on the basis of (3.6), can be expressed by means of the equality

$$
\breve{\mathbf{I}}^{t}\left(\tau, \mathbf{E}_{r_{1}}^{t}\right)=\breve{\mathbf{I}}^{t}\left(\tau, \mathbf{E}_{r_{2}}^{t}\right) \quad \forall \tau \in \mathbb{R}^{++} .
$$

Hence, it follows that the quantity $\breve{\mathbf{I}}^{t}$ can be assumed to characterize an equivalence class $\sigma_{\mathcal{R}} \in \Sigma_{\mathcal{R}}$ and, therefore, is a minimal state.

4. Thermodynamics and free energies. The central role that thermodynamics plays in the study of any physical problem is well known. For this purpose it is fundamental to determine the most appropriate norms, the choice of which in the linear case is strongly related to the various free energies of the material.

We begin by defining the work done by a process $P$ of duration $d$ and applied at time $t>0$ to an initial state $\sigma(t)$ as

$$
W(\sigma, P)=\int_{0}^{d} \mathbf{T}(\sigma(\tau), P(\tau)) \cdot \mathbf{L}^{P}(\tau) d \tau,
$$

where $\mathbf{L}^{P}$ determines $P$ as in (3.1) and $\sigma(\tau)$ belongs to the set of states which can be obtained by starting from $\sigma(t)$ with a suitable process, that is,

$$
\Sigma_{\sigma}=\left\{\sigma^{\prime} \in \Sigma ; \exists P \in \Pi, \sigma^{\prime}=\rho(\sigma, P)\right\} .
$$

Then, since in this article we are concerned with isothermal processes, we recall the Second Law of Thermodynamics in the traditional form of the Clausius-Duhem inequality.

Clausius-Duhem's inequality (isothermal processes). The work done on any cycle $(\sigma, P)$ is non-negative, i.e.

$$
W(\sigma, P)=\oint_{0}^{d} \mathbf{T}(\sigma(\tau), P(\tau)) \cdot \mathbf{L}^{P}(\tau) d \tau \geq 0
$$

with equality corresponding to reversible processes.

We now give two useful definitions. 
Definition 2. A subset $S \subset \Sigma$ is called invariant under $\rho$ if, for every $\sigma^{i} \in \Sigma$ and $P \in \Pi$, the state $\sigma=\rho\left(\sigma^{i}, P\right) \in S$.

Definition 3. A state $\sigma \in \Sigma$ is attainable from all of $\Sigma$ if, for any initial state $\sigma^{i}$, there exists a process $P \in \Pi$ such that $\sigma=\rho\left(\sigma^{i}, P\right)$. If any $\sigma$ is attainable from every other state $\sigma^{\prime} \in \Sigma$, the simple material system is attainable.

We observe that, for simple material systems with fading memory, not all states are attainable; moreover, in particular, cycles are quite rare. Therefore, following [15], we assume a different and more restrictive formulation of the Second Law.

For this purpose we denote by

$$
\mathcal{W}(\sigma)=\{W(\sigma, P) ; P \in \Pi\}
$$

the set of values of work done upon applying to a given state $\sigma \in \Sigma$ all the possible processes $P \in \Pi$; the final state induced by each of these applied processes is any state $\sigma^{\prime} \in \Sigma_{\sigma}$ defined in (4.2).

Strong Dissipation Principle. The set $\mathcal{W}(\sigma)$ is bounded below for all $\sigma \in \Sigma$; moreover, there exists a state $\sigma^{\dagger}$, called the zero state, such that

$$
\inf \mathcal{W}\left(\sigma^{\dagger}\right)=0 .
$$

To avoid a contradiction with the Second Law, the assumption of this Strong Dissipation Principle requires a modification of the set (3.13) of states as follows:

$$
\Sigma=\left\{\sigma \in \Gamma_{r} ; \inf \mathcal{W}(\sigma)>-\infty\right\} .
$$

In fact, if condition (4.6) were not true, then the work $-W(\sigma, P)$ given by the material would be unbounded from above; consequently, as $P$ varies, we could extract infinite energy, thus generating a perpetual motion.

We now introduce the notion of free energy.

Definition 4. A function $\psi: S_{\psi} \rightarrow \mathbb{R}^{+}$is a free energy if

(a) the domain $S_{\psi} \subset \Sigma$ is invariant under $\rho$,

(b) for any pair $\sigma_{1}, \sigma_{2} \in S_{\psi}$ and for every $P \in \Pi$ such that $\sigma_{2}=\rho\left(\sigma_{1}, P\right)$, we have

$$
\psi\left(\sigma_{2}\right)-\psi\left(\sigma_{1}\right) \leq W\left(\sigma_{1}, P\right) .
$$

The free energy, the existence of which was proved as a consequence of the Second Law of Thermodynamics expressed by (4.3), does not have a unique expression for materials with memory ([7, [16] and [24]). In fact, there are many free energies, making up a convex set with a minimum and a maximum element, denoted by $\psi_{m}$ and $\psi_{M}$, respectively.

Definition 5. A functional $\psi_{m}$ is called the minimum free energy if

i) $\psi_{m}$ is a free energy with domain $\mathcal{S}_{\psi}=\Sigma$,

ii) the zero state $\sigma^{\dagger} \in \Sigma$ is such that $\psi_{m}\left(\sigma^{\dagger}\right)=0$,

iii) for any free energy $\psi: \mathcal{S}_{\psi} \rightarrow \mathbb{R}^{+}$such that $\sigma^{\dagger} \in \mathcal{S}_{\psi}$ and $\psi\left(\sigma^{\dagger}\right)=0$, we have

$$
\psi(\sigma) \geq \psi_{m}(\sigma) \quad \forall \sigma \in \mathcal{S}_{\psi} .
$$

REMARK 1. The minimum free energy, if it exists, is unique.

Proof. If two such energies $\psi_{m_{1}}$ and $\psi_{m_{2}}$ were to exist, then, for any $\sigma \in \mathcal{S}_{\psi}$, from (4.8) we would have $\psi_{m_{1}}(\sigma) \geq \psi_{m_{2}}(\sigma)$ by considering $\psi_{m_{2}}(\sigma)$ as the minimum and, analogously, also $\psi_{m_{2}}(\sigma) \geq \psi_{m_{1}}(\sigma)$. Hence, $\psi_{m_{1}}(\sigma)=\psi_{m_{2}}(\sigma)$ for any $\sigma \in \mathcal{S}_{\psi}$. 
The characterization of the minimum free energy is given by the following theorem [15.

THEOREM 1. The functional

$$
\psi_{m}(\sigma):=-\inf \mathcal{W}(\sigma):=N(\sigma)
$$

is the minimum free energy.

Let $\sigma_{0}, \sigma \in \Sigma$ be any pair of states such that $\sigma \in \Sigma_{\sigma_{0}}$, as defined in (4.2); we consider the set

$$
N\left(\sigma_{0}, \sigma\right)=\left\{W\left(\sigma_{0}, P\right) ; \quad \forall P \in \Pi, \rho\left(\sigma_{0}, P\right)=\sigma\right\} .
$$

The Strong Dissipation Principle yields that this set is bounded below.

We recall the result expressed by the following theorem proved in [17.

Theorem 2. For any fixed $\sigma^{i}$, the functional $\psi_{M}^{\sigma^{i}}: \Sigma_{\sigma^{i}} \rightarrow \mathbb{R}^{+}$defined by

$$
\psi_{M}^{\sigma^{i}}(\sigma)=\inf N\left(\sigma^{i} ; \sigma\right)+\psi_{m}\left(\sigma^{i}\right)
$$

is a free energy, called a maximum free energy. For any free energy $\psi: \mathcal{S}_{\psi} \rightarrow \mathbb{R}^{+}$such that $\mathcal{S}_{\psi} \supset \Sigma_{\sigma^{i}}$ and $\psi\left(\sigma^{i}\right)=\psi_{m}\left(\sigma^{i}\right)$, we have

$$
\psi(\sigma) \leq \psi_{M}^{\sigma^{i}}(\sigma), \quad \forall \sigma \in \Sigma_{\sigma^{i}} .
$$

From the expression (4.1) for the work and the inequality (4.7) which characterizes a free energy, an important inequality follows at once.

Under the hypothesis that the process be continuous at any time $t$, we obtain

$$
\dot{\psi}(\sigma(t)) \leq \hat{\mathbf{T}}(\sigma(t), P(t)) \cdot \mathbf{L}(t) .
$$

This inequality, upon taking into account the constitutive equation (2.1) for the fluid under consideration and (3.4) in the case where $P$ is applied at time $t=0$ so that $\tau \equiv t$, becomes

$$
\dot{\psi}(t) \leq \mathbf{T}\left(\mathbf{E}_{r}^{t}\right) \cdot \dot{\mathbf{E}}(t) .
$$

Consequently, we can also consider the equality

$$
\dot{\psi}(t)+D(t)=\mathbf{T}(t) \cdot \dot{\mathbf{E}}(t),
$$

where we have introduced $D(t)$, called the internal dissipation function, which must be non-negative because of the Second Law.

5. Work and w-equivalence between states. It is interesting to give expressions for the work corresponding to some important cases for linear viscoelastic fluids [2].

A first expression is related to the work done on the fluid by the relative strain history up to time $t$ when the final state is $\sigma(t)=\mathbf{E}_{r}^{t}$.

Using (4.1), the constitutive equation (2.1) for the stress tensor and the condition

$$
\mathbf{I} \cdot \dot{\mathbf{E}}=\nabla \cdot \mathbf{v}=0,
$$

which follows from the constraint of incompressibility, the work in this case is given by

$$
\tilde{W}\left(\mathbf{E}_{r}^{t}\right)=\int_{-\infty}^{t} \mathbf{T}(\tau) \cdot \dot{\mathbf{E}}(\tau) d \tau=2 \int_{-\infty}^{t} \int_{0}^{+\infty} \mu^{\prime}(s) \mathbf{E}_{r}^{\tau}(s) d s \cdot \dot{\mathbf{E}}(\tau) d \tau<+\infty,
$$


since we consider only relative strain histories which give finite work.

Under the hypothesis of an even extension of $\mu(s)$, by using suitable integrations by parts and (2.2), we can give (5.2) 2 the following forms:

$$
\begin{aligned}
\tilde{W}\left(\mathbf{E}_{r}^{t}\right) & =\int_{-\infty}^{t} \int_{-\infty}^{t} \mu(|\rho-u|) \dot{\mathbf{E}}(u) \cdot \dot{\mathbf{E}}(\rho) d u d \rho \\
& =\int_{0}^{+\infty} \int_{0}^{+\infty} \mu(|\eta-\xi|) \dot{\mathbf{E}}^{t}(\xi) \cdot \dot{\mathbf{E}}^{t}(\eta) d \xi d \eta \\
& =\int_{0}^{+\infty} \int_{0}^{+\infty} \mu_{12}(|\eta-\xi|) \mathbf{E}_{r}^{t}(\xi) \cdot \mathbf{E}_{r}^{t}(\eta) d \xi d \eta
\end{aligned}
$$

where

$$
\mu_{12}(|\eta-\xi|)=\frac{\partial^{2}}{\partial \eta \partial \xi} \mu(|\eta-\xi|)=-\mu^{\prime \prime}(|\eta-\xi|)-2 \delta(\eta-\xi) \mu^{\prime}(|\eta-\xi|) .
$$

Moreover, by virtue of Plancherel's theorem and $(2.15) 2$, in the frequency domain we also have

$$
\tilde{W}\left(\mathbf{E}_{r}^{t}\right)=-\frac{1}{\pi} \int_{-\infty}^{+\infty} \omega \mu_{s}^{\prime}(\omega) \mathbf{E}_{r+}^{t}(\omega) \cdot\left[\mathbf{E}_{r+}^{t}(\omega)\right]^{*} d \omega .
$$

Using this expression and the restriction (2.16), we have the following result [2].

Lemma 3 . The work done on the material by the relative strain history $\mathbf{E}_{r}^{t}(s) \forall s \in \mathbb{R}^{++}$ is a non-negative quantity.

We now consider the work done in the general case where the process $P(\tau)=\dot{\mathbf{E}}_{P}(\tau)$ $\forall \tau \in[0, d)$ is applied to the state $\sigma(t)=\mathbf{E}_{r}^{t}(s) \forall s \in \mathbb{R}^{++}$at time $t>0$. Using (4.1), (3.5) and (3.6), we have

$$
W(\sigma, P)=\int_{0}^{d}\left[2 \int_{0}^{\tau} \mu(\tau-\xi) \dot{\mathbf{E}}_{P}(\xi) d \xi-\breve{\mathbf{I}}^{t}\left(\tau, \mathbf{E}_{r}^{t}\right)\right] \cdot \dot{\mathbf{E}}_{P}(\tau) d \tau
$$

We observe that in such an expression the duration of the process generally has a finite value, $d<+\infty$. However, it is useful to consider the process $P$ as being defined on $\mathbb{R}^{+}$by taking its trivial extension, $P(\tau)=\mathbf{0} \forall \tau \in[d,+\infty)$.

Thus, the expression (5.6), where $(\sigma, P)=\left(\mathbf{E}_{r}^{t}, \dot{\mathbf{E}}_{P}\right)$ can be replaced by $\left(\breve{\mathbf{I}}^{t}, \dot{\mathbf{E}}_{P}\right)$ upon extending $P$ to $\mathbb{R}^{+}$by means of its trivial extension, can be written as

$$
\begin{aligned}
& W\left(\breve{\mathbf{I}}^{t}, \dot{\mathbf{E}}_{P}\right)=\int_{0}^{+\infty}\left[\int_{0}^{+\infty} \mu(|\tau-\xi|) \dot{\mathbf{E}}_{P}(\xi) d \xi-\breve{\mathbf{I}}^{t}\left(\tau, \mathbf{E}_{r}^{t}\right)\right] \cdot \dot{\mathbf{E}}_{P}(\tau) d \tau \\
& =\frac{1}{\pi} \int_{-\infty}^{+\infty} \mu_{c}(\omega) \dot{\mathbf{E}}_{P+}(\omega) \cdot\left(\dot{\mathbf{E}}_{P+}(\omega)\right)^{*} d \omega-\frac{1}{2 \pi} \int_{-\infty}^{+\infty} \breve{\mathbf{I}}_{+}^{t}(\omega) \cdot\left[\dot{\mathbf{E}}_{P+}(\omega)\right]^{*} d \omega,
\end{aligned}
$$

where we have applied Plancherel's theorem and denoted by $\breve{\mathbf{I}}_{+}^{t}(\omega)$ the Fourier transform of $\breve{\mathbf{I}}^{t}\left(\tau, \mathbf{E}_{r}^{t}\right)$.

We now consider the particular case where $P$ is applied at time $t=0$, when the initial state is $\sigma(0)=\mathbf{E}_{r}^{0}(s)$, and again extended to $\mathbb{R}^{+}$as before.

Taking into account the expression (3.7) for $\mathbf{T}$, (5.6) becomes

$$
\tilde{W}\left(\mathbf{E}_{r}^{0}, \dot{\mathbf{E}}_{P}\right)=\int_{0}^{+\infty}\left[\int_{0}^{+\infty} \mu(|t-\tau|) \dot{\mathbf{E}}_{P}(\tau) d \tau-\breve{\mathbf{I}}^{0}\left(t, \mathbf{E}_{r}^{0}\right)\right] \cdot \dot{\mathbf{E}}_{P}(t) d t .
$$


Moreover, if the initial relative history is the zero relative history, $\mathbf{E}_{r}^{0}(s)=\mathbf{0}_{r}^{\dagger}(s)=\mathbf{0}$ $\forall s \in \mathbb{R}^{++}$, then by using (3.8) we can evaluate the work due only to the process, obtaining

$$
\tilde{W}\left(\mathbf{0}_{r}^{\dagger}, \dot{\mathbf{E}}_{P}\right)=\int_{0}^{+\infty} \int_{0}^{+\infty} \mu(|t-\tau|) \dot{\mathbf{E}}_{P}(\tau) \cdot \dot{\mathbf{E}}_{P}(t) d \tau d t .
$$

Following Gentili [20, we introduce the following definition.

Definition 6. A process $P$, of any duration, is said to be a finite work process if

$$
\tilde{W}\left(\mathbf{0}_{r}^{\dagger}, \dot{\mathbf{E}}_{P}\right)<+\infty
$$

In 2 we showed that

$$
\tilde{W}\left(\mathbf{0}_{r}^{\dagger}, \dot{\mathbf{E}}_{P}\right)=\frac{1}{\pi} \int_{-\infty}^{+\infty} \mu_{c}(\omega) \dot{\mathbf{E}}_{P+}(\omega) \cdot\left[\dot{\mathbf{E}}_{P+}(\omega)\right]^{*} d \omega>0
$$

where we have used Plancherel's theorem, $(2.9)_{2}$, by virtue of (2.12).

The space of the processes can be defined as follows [20]:

$$
\tilde{H}_{\mu}\left(\mathbb{R}^{+}\right)=\left\{\dot{\mathbf{E}}_{P}: \mathbb{R}^{+} \rightarrow \operatorname{Sym} ; \int_{-\infty}^{+\infty} \mu_{c}(\omega) \dot{\mathbf{E}}_{P+}(\omega) \cdot\left[\dot{\mathbf{E}}_{P+}(\omega)\right]^{*} d \omega<+\infty\right\} .
$$

This space, by means of a completion with the norm corresponding to the inner product $\left(\mathbf{w}_{1}, \mathbf{w}_{2}\right)_{\mu}=\int_{-\infty}^{+\infty} \mu_{c}(\omega) \mathbf{w}_{1+}(\omega) \cdot\left[\mathbf{w}_{2+}(\omega)\right]^{*} d \omega$, yields the Hilbert space $H_{\mu}\left(\mathbb{R}^{+}\right)[2]$.

The dual of the space $H_{\mu}\left(\mathbb{R}^{+}\right)$is

$$
H_{\mu}^{\prime}\left(\mathbb{R}^{+}\right)=\left\{\breve{\mathbf{I}}^{t}: \mathbb{R}^{+} \rightarrow \operatorname{Sym} ; \int_{-\infty}^{+\infty} \breve{\mathbf{I}}_{+}^{t}(\omega) \cdot\left[\dot{\mathbf{E}}_{P+}(\omega)\right]^{*} d \omega<+\infty \quad \forall \dot{\mathbf{E}}_{P} \in H_{\mu}\left(\mathbb{R}^{+}\right)\right\}
$$

and characterizes the possible states of our fluid.

Another definition of equivalence between two states of the fluid can be given in terms of the work, as has been done for viscoelastic solids [20].

Definition 7. Two states $\sigma_{j}(t)=\mathbf{E}_{r_{j}}^{t}(j=1,2)$ are said to be w-equivalent if for every $P:[0, \tau) \rightarrow$ Sym and for every $\tau>0$ they satisfy

$$
\tilde{W}\left(\mathbf{E}_{r_{1}}^{t}, \dot{\mathbf{E}}_{P}\right)=\tilde{W}\left(\mathbf{E}_{r_{2}}^{t}, \dot{\mathbf{E}}_{P}\right) .
$$

In 2] we showed that Definitions 1 and 7 coincide by virtue of the following lemma.

Lemma 4. For every fluid characterized by the constitutive equation (2.1), two states are w-equivalent if and only if they are equivalent in the sense of Definition 1.

The equivalence of Definitions 1 and 7 allows us to consider a unique equivalence relation $\mathcal{R}$ in $\Sigma$ and, consequently, only one quotient space $\Sigma_{\mathcal{R}}$. The equivalence classes $\sigma_{\mathcal{R}}$ of $\mathcal{R}$ characterize the states which are indistinguishable both because they give the same extra stress and since we obtain the same work by starting from each of them and applying any process with an arbitrary duration.

Therefore, we can express the minimum and the maximum free energy in terms of $\sigma_{\mathcal{R}} \in \Sigma_{\mathcal{R}}$, by virtue of the w-equivalence, as the following two remarks state. 
REMARK 2. It is always possible to represent the minimum free energy as a function of the minimal state $\sigma_{R}$. From the definition of $\mathcal{W}(\sigma)$ given by (4.4) and from the fact that $W(\sigma, P)=W\left(\sigma_{R}, P\right)$ for all $P \in \Pi$, it is clear that

$$
\inf \mathcal{W}(\sigma)=\inf \tilde{\mathcal{W}}\left(\sigma_{R}\right) .
$$

Hence, if $\sigma \in \sigma_{R}$ we have

$$
\psi_{m}(\sigma)=\psi_{m}\left(\sigma_{R}\right)
$$

therefore, the minimum free energy is independent of the representation of state which is used.

REMARK 3. From any $\sigma^{i} \in \Sigma$ we may obtain a different free energy. Moreover, for a fixed $\sigma^{i} \in \Sigma$ the definition of maximum free energy may depend on the definition of state. We can, however, construct a maximum free energy that is defined on the space of minimal states. In other words, if we consider the definition of minimal state, then (4.10) is replaced by

$$
N\left(\sigma_{0 R}, \sigma_{R}\right)=\left\{W\left(\sigma_{0 R}, P\right) ; \forall P \in \Pi, \rho\left(\sigma_{0 R}, P\right)=\sigma_{R}\right\} .
$$

Since this set is generally larger than $N\left(\sigma_{0}, \sigma\right)$, if $\sigma_{0} \in \sigma_{0 R}$ and $\sigma \in \sigma_{R}$, the maximum free energy, defined on $\Sigma_{R}$ as

$$
\psi_{M}^{\sigma_{R}^{i}}\left(\sigma_{R}\right)=\inf N\left(\sigma_{R}^{i}, \sigma_{R}\right)+\psi_{m}\left(\sigma_{R}^{i}\right),
$$

satisfies the inequality

$$
\psi_{M}^{\sigma_{R}^{i}}\left(\sigma_{R}\right) \leq \psi_{M}^{\sigma^{i}}(\sigma), \quad \sigma^{i} \in \sigma_{R}^{i}, \sigma \in \sigma_{R}
$$

Relation (4.12) will apply to any free energy $\psi\left(\sigma_{R}\right)$ defined on $\Sigma_{R}$ provided that $\psi\left(\sigma_{R}^{i}\right)=\psi_{m}\left(\sigma_{R}^{i}\right)$.

6. Free energies. The free energies, which have been introduced for linear viscoelastic solids, can be defined also for linear viscoelastic fluids with a few changes. Since some of these energies have already been derived in [3], we shall refer to this article and only recall the relevant results.

6.1. Graffi-Volterra's free energy. We firstly consider the free energy expressed by the Graffi-Volterra functiona 1 (see [22, 23] and [32]). This expression is very important because it is frequently used in applications.

For the fluids we are considering, the corresponding functional, derived in [3], is

$$
\psi_{G}(t)=-\int_{0}^{+\infty} \mu^{\prime}(s) \mathbf{E}_{r}^{t}(s) \cdot \mathbf{E}_{r}^{t}(s) d s ;
$$

1 The expression for this free energy corresponding to viscoelastic solids is

$$
\psi_{G}\left(\mathbf{E}^{t}\right)=\frac{1}{2} \mathbf{E}(t) \cdot \mathbf{G}_{\infty} \mathbf{E}(t)-\frac{1}{2} \int_{0}^{+\infty} \mathbf{E}_{r}^{t}(\xi) \cdot \mathbf{G}^{\prime}(\xi) \mathbf{E}_{r}^{t}(\xi) d \xi,
$$

where the relaxation function $\mathbf{G}$ is a fourth-order tensor which is assumed to be symmetric, while the symmetry of $\mathbf{G}_{\infty}=\lim _{s \rightarrow+\infty} \mathbf{G}(s)$ is required by the thermodynamic laws; moreover, $\mathbf{G}^{\prime}$ is negative definite, $\mathbf{G}^{\prime}(s)<0$, while $\mathbf{G}_{\infty}$ and $\mathbf{G}^{\prime \prime}$ are such that $\mathbf{G}_{\infty}>0$ and $\mathbf{G}^{\prime \prime}(s) \geq 0 \forall s \in \mathbb{R}^{+}$. This is a generalization of a free energy functional determined by Graffi and is called the Graffi-Volterra free energy. 
this gives a free energy upon assuming that $\mu^{\prime}(s) \leq 0$ and $\mu^{\prime \prime}(s) \geq 0 \forall s \in \mathbb{R}^{+}$. We note that these restrictions coincide with those considered by Slemrod in [29] while studying stability problems for these fluids (see [30, 25] and [26]), by virtue of the assumptions on $\mu$ defined in (2.3), viz.

$$
\lim _{s \rightarrow+\infty} \mu(s)=0, \quad \mu(s)>0 .
$$

In order to derive the internal dissipation, it is enough to evaluate the time derivative of (6.1), by taking into account the definition of $\mathbf{E}_{r}^{t}$, the constitutive equation (2.1) and the constraint of incompressibility (5.1). Thus, in 3] it was proved that (4.15) is satisfied by (6.1) with the following internal dissipation:

$$
D_{G}(t)=\int_{0}^{+\infty} \mu^{\prime \prime}(s) \mathbf{E}_{r}^{t}(s) \cdot \mathbf{E}_{r}^{t}(s) d s \geq 0,
$$

which is a non-negative quantity for all histories because of the hypothesis on the second derivative of $\mu(s)$.

6.2. Day's free energy. Another free energy already introduced for viscoelastic solids is given by Day's functiona 2 [ . For our viscoelastic fluids such a functional becomes

$$
\psi_{\text {Day }}(t)=\frac{1}{\mu_{0}}\left[\int_{0}^{+\infty} \mu^{\prime}(s) \mathbf{E}_{r}^{t}(s) d s\right]^{2},
$$

where $\mu_{0}=\mu(0)>0$, by virtue of $(2.19)_{1}$.

Taking the time derivative of this function and performing an integration by parts, using the definition (2.2) of $\mathbf{E}_{r}^{t}$ together with the constitutive equation (2.1) and the constraint of incompressibility (5.1), yields

$$
\begin{gathered}
\dot{\psi}_{\text {Day }}(t)=\frac{2}{\mu_{0}} \int_{0}^{+\infty} \mu^{\prime}(s) \mathbf{E}_{r}^{t}(s) d s \cdot \int_{0}^{+\infty} \mu^{\prime}(s)\left[\dot{\mathbf{E}}^{t}(s)-\dot{\mathbf{E}}(t)\right] d s \\
=\frac{2}{\mu_{0}} \int_{0}^{+\infty} \mu^{\prime}(s) \mathbf{E}_{r}^{t}(s) d s \cdot\left[-\int_{0}^{+\infty} \mu^{\prime}(s) \frac{d}{d s} \mathbf{E}^{t}(s) d s+\mu_{0} \dot{\mathbf{E}}(t)\right] \\
=\mathbf{T}(t) \cdot \dot{\mathbf{E}}(t)+\frac{2}{\mu_{0}} \int_{0}^{+\infty} \mu^{\prime}(s) \mathbf{E}_{r}^{t}(s) d s \cdot \int_{0}^{+\infty} \mu^{\prime \prime}(s) \mathbf{E}_{r}^{t}(s) d s
\end{gathered}
$$

The negative of the last term of this relation must give the corresponding non-negative dissipation $D_{D a y}(t)$.

We now assume the exponential form required for Day's free energy, by putting

$$
\mu^{\prime}(s)=\mu^{\prime}(0) e^{-\gamma s}, \quad \gamma>0,
$$

such that $\mu^{\prime}(0)<0$, that is, satisfying $(2.20)_{3}$. Thus, we have

$$
\mu^{\prime \prime}(s)=-\gamma \mu^{\prime}(s),
$$

${ }^{2}$ The functional

$$
\psi_{D a y}\left(\mathbf{E}^{t}\right)=\frac{1}{2} \mathbf{E}(t) \cdot \mathbf{G}_{\infty} \mathbf{E}(t)+\frac{1}{2}\left[\left(\mathbf{G}_{0}-\mathbf{G}_{\infty}\right)^{-1 / 2} \int_{0}^{+\infty} \mathbf{G}^{\prime}(s) \mathbf{E}_{r}^{t}(s) d s\right]^{2}
$$

represents the general expression for the Day free energy (see [19] ch. 3), which has been proposed for viscoelastic solids. In this form, in particular, the fourth-order tensors $\mathbf{G}_{0}$ and $\mathbf{G}_{\infty}$ are such that $\mathbf{G}_{0}-\mathbf{G}_{\infty}>0$, while the fourth-order tensor $\mathbf{G}^{\prime}(s)$ is isotropic and has an exponential form. 
which allows us to write (6.5) as

$$
\dot{\psi}_{D a y}(t)=\mathbf{T}(t) \cdot \dot{\mathbf{E}}(t)-D_{D a y}(t)
$$

where the internal dissipation $D_{D a y}(t)$ is given by

$$
D_{\text {Day }}(t)=\frac{2 \gamma}{\mu_{0}}\left[\int_{0}^{+\infty} \mu^{\prime}(s) \mathbf{E}_{r}^{t}(s) d s\right]^{2} \geq 0 .
$$

6.3. Dill's free energy. Also the Dill functiona $\sqrt{3}$, proposed for viscoelastic solids in [12, can be considered for our fluids in the following form:

$$
\psi_{\text {Dill }}(t)=\int_{0}^{+\infty} \int_{0}^{+\infty} \mu^{\prime \prime}\left(\xi_{1}+\xi_{2}\right) \mathbf{E}_{r}^{t}\left(\xi_{1}\right) \cdot \mathbf{E}_{r}^{t}\left(\xi_{2}\right) d \xi_{1} d \xi_{2} .
$$

The requirement that $\psi(t)$ be non-negative for all $t$ implies that the kernel $\mu^{\prime \prime}\left(\xi_{1}+\xi_{2}\right)$ must be such that the integral is non-negative for all relative histories.

Taking into account the definition (2.2) of $\mathbf{E}_{r}^{t}$ the constitutive equation (2.1) and the constraint of incompressibility (5.1), the time derivative of (6.10), after some integrations, becomes

$$
\begin{aligned}
\dot{\psi}_{\text {Dill }}(t)= & \int_{0}^{+\infty} \int_{0}^{+\infty} \mu^{\prime \prime}\left(\xi_{1}+\xi_{2}\right)\left\{\left[\dot{\mathbf{E}}^{t}\left(\xi_{1}\right)-\dot{\mathbf{E}}(t)\right] \cdot\left[\mathbf{E}^{t}\left(\xi_{2}\right)-\mathbf{E}(t)\right]\right. \\
& \left.+\left[\mathbf{E}^{t}\left(\xi_{1}\right)-\mathbf{E}(t)\right] \cdot\left[\dot{\mathbf{E}}^{t}\left(\xi_{2}\right)-\dot{\mathbf{E}}(t)\right]\right\} d \xi_{1} d \xi_{2} \\
= & {\left[2 \mu_{0} \mathbf{E}(t)+2 \int_{0}^{+\infty} \mu^{\prime}(s) \mathbf{E}^{t}(s) d s\right] \cdot \dot{\mathbf{E}}(t) } \\
& +2 \int_{0}^{+\infty} \int_{0}^{+\infty} \mu^{\prime}\left(\xi_{1}+\xi_{2}\right) \dot{\mathbf{E}}^{t}\left(\xi_{1}\right) \cdot \dot{\mathbf{E}}^{t}\left(\xi_{2}\right) d \xi_{1} d \xi_{2} \\
= & \mathbf{T}(t) \cdot \dot{\mathbf{E}}(t)+2 \int_{0}^{+\infty} \int_{0}^{+\infty} \mu^{\prime}\left(\xi_{1}+\xi_{2}\right) \dot{\mathbf{E}}^{t}\left(\xi_{1}\right) \cdot \dot{\mathbf{E}}^{t}\left(\xi_{2}\right) d \xi_{1} d \xi_{2} .
\end{aligned}
$$

Hence, it follows that now (4.15) is still satisfied if the kernel $\mu^{\prime}$ is such that we have a non-negative dissipation, given by

$$
D_{D i l l}(t)=-2 \int_{0}^{+\infty} \int_{0}^{+\infty} \mu^{\prime}\left(\xi_{1}+\xi_{2}\right) \dot{\mathbf{E}}^{t}\left(\xi_{1}\right) \cdot \dot{\mathbf{E}}^{t}\left(\xi_{2}\right) d \xi_{1} d \xi_{2} \geq 0 .
$$

6.4. Fabrizio's free energy. A new free energy introduced for viscoelastic solids is the Fabrizio functional 4 considered, in particular, in [13] and [10, where it is denoted by

\footnotetext{
${ }^{3}$ Another example of free energy is the Dill free energy, which, for viscoelastic solids, has the following expression:

$$
\psi_{D i l l}\left(\mathbf{E}^{t}\right)=\frac{1}{2} \mathbf{E}(t) \cdot \mathbf{G}_{\infty} \mathbf{E}(t)+\frac{1}{2} \int_{0}^{+\infty} \int_{0}^{+\infty} \mathbf{E}_{r}^{t}\left(\xi_{1}\right) \cdot \mathbf{G}^{\prime \prime}\left(\xi_{1}+\xi_{2}\right) \mathbf{E}_{r}^{t}\left(\xi_{2}\right) d \xi_{1} d \xi_{2},
$$

where the fourth-order tensors $\mathbf{G}_{\infty}$ and $\mathbf{G}^{\prime \prime}$ satisfy the usual conditions, $\mathbf{G}_{\infty}>0$ and $\mathbf{G}^{\prime \prime}(s) \geq 0$ $\forall s \in \mathbb{R}^{+}$.

4 A new functional which has been recently proposed as a free energy for viscoelastic solids is the Fabrizio free energy, which has the form

$$
\psi_{F}\left(\breve{\mathbf{I}}^{t}\right)=\frac{1}{2} \mathbf{E}(t) \cdot \mathbf{G}_{\infty} \mathbf{E}(t)-\frac{1}{2} \int_{0}^{+\infty} \dot{\mathbf{G}}^{-1}(\tau) \breve{\mathbf{I}}_{(1)}^{t}(\tau) \cdot \breve{\mathbf{I}}_{(1)}^{t}(\tau) d \tau
$$

In this expression $\dot{\mathbf{G}}^{-1}(\tau)$ is the inverse tensor of the fourth-order tensor $\dot{\mathbf{G}}(\tau) \equiv \mathbf{G}^{\prime}(\tau)$ and $\breve{\mathbf{I}}_{(1)}^{t}(\tau)$ denotes the derivative with respect to $\tau$ of $\breve{\mathbf{I}}^{t}\left(\tau, \mathbf{E}_{r}^{t}\right)=-\int_{0}^{+\infty} \dot{\mathbf{G}}(s+\tau) \mathbf{E}_{r}^{t}(s) d s$, which characterizes the
} 
$\psi_{F}$. The form assumed by such a functional for our fluids, already derived in [3], is given by

$$
\psi_{F}(t)=-\frac{1}{4} \int_{0}^{+\infty} \frac{1}{\mu^{\prime}(\tau)} \breve{\mathbf{I}}_{(1)}^{t}(\tau) \cdot \breve{\mathbf{I}}_{(1)}^{t}(\tau) d \tau
$$

where we have denoted by $\breve{\mathbf{I}}_{(1)}^{t}(\tau)$ the derivative with respect to $\tau$ of $\breve{\mathbf{I}}^{t}\left(\tau, \mathbf{E}_{r}^{t}\right)$ introduced in (3.6), that is,

$$
\breve{\mathbf{I}}_{(1)}^{t}(\tau)=\frac{d}{d \tau} \breve{\mathbf{I}}^{t}\left(\tau, \mathbf{E}_{r}^{t}\right)=-2 \int_{0}^{+\infty} \mu^{\prime \prime}(\xi+\tau) \mathbf{E}_{r}^{t}(\xi) d \xi .
$$

Since the functional (6.13) is to give a non-negative quantity, now we must still assume that $\mu^{\prime \prime}(\tau)$ is a non-negative function of $\tau \in \mathbb{R}^{+}$and consequently that $\mu^{\prime}(\tau)$ is a nonpositive function of $\tau \in \mathbb{R}^{+}$.

As was already observed in [3], we note that no problem arises from the presence of $1 / \mu^{\prime}(\tau)$, which diverges as $\tau$ tends to infinity, because this factor is multiplied by other factors (see (6.37) below) which assure the existence of the integral under consideration. Moreover, the domain of definition of the functional $\psi_{F}$ is characterized by the following space of functions:

$$
H_{F}^{\prime}\left(\mathbb{R}^{+}\right)=\left\{\breve{\mathbf{I}}^{t} ;\left|\int_{0}^{+\infty} \frac{1}{\mu^{\prime}(\tau)} \breve{\mathbf{I}}_{(1)}^{t}(\tau) \cdot \breve{\mathbf{I}}_{(1)}^{t}(\tau) d \tau\right|<+\infty\right\},
$$

which yields a much larger space than that corresponding to the Graffi-Volterra free energy $\psi_{G}$.

In order to prove that $\psi_{F}$ is a free energy, in $\left[3\right.$ the derivative of $\psi_{F}(t)$,

$$
\dot{\psi}_{F}(t)=-\frac{1}{2} \int_{0}^{+\infty} \frac{1}{\mu^{\prime}(\tau)} \dot{\mathbf{I}}_{(1)}^{t}(\tau) \cdot \breve{\mathbf{I}}_{(1)}^{t}(\tau) d \tau
$$

was evaluated by means of (6.14) and the relations

$$
\dot{\breve{\mathbf{I}}}_{(1)}^{t}(\tau)=\frac{d}{d t} \breve{\mathbf{I}}_{(1)}^{t}(\tau)=\breve{\mathbf{I}}_{(2)}^{t}(\tau)-2 \mu^{\prime}(\tau) \dot{\mathbf{E}}(t),
$$

where

$$
\breve{\mathbf{I}}_{(2)}^{t}(\tau)=\frac{d^{2}}{d \tau^{2}} \breve{\mathbf{I}}^{t}(\tau)=\frac{d}{d \tau} \breve{\mathbf{I}}_{(1)}^{t}(\tau)
$$

and

$$
\int_{0}^{+\infty} \breve{\mathbf{I}}_{(1)}^{t}(\tau) d \tau=2 \int_{0}^{+\infty} \mu^{\prime}(\xi) \mathbf{E}_{r}^{t}(\xi) d \xi=\mathbf{T}(t)+p(t) \mathbf{I} .
$$

Thus, in 3 the expression

$$
\dot{\psi}_{F}(t)=\mathbf{T}(t) \cdot \dot{\mathbf{E}}(t)-D_{F}(t)
$$

was derived, with

$$
D_{F}(t)=-\frac{1}{4} \frac{1}{\mu^{\prime}(0)} \breve{\mathbf{I}}_{(1)}^{t}(0) \cdot \breve{\mathbf{I}}_{(1)}^{t}(0)-\frac{1}{4} \int_{0}^{+\infty} \frac{d}{d \tau}\left[\frac{1}{\mu^{\prime}(\tau)}\right] \breve{\mathbf{I}}_{(1)}^{t}(\tau) \cdot \breve{\mathbf{I}}_{(1)}^{t}(\tau) d \tau \geq 0 .
$$

minimal state for the material. The assumption that $\ddot{\mathbf{G}}(\tau) \equiv \mathbf{G}^{\prime \prime}(\tau)$ be a positive semidefinite tensor for all $\tau \in \mathbb{R}^{+}$yields a non-negative term for the integral present in this expression, since $\dot{\mathbf{G}}(\tau)$ is negative semidefinite for all $\tau \in \mathbb{R}^{+}$. 
This last expression is a non-negative quantity because of the properties of $\mu$,

$$
\frac{d}{d \tau}\left[\frac{1}{\mu^{\prime}(\tau)}\right]=-\frac{\mu^{\prime \prime}(\tau)}{\left[\mu^{\prime}(\tau)\right]^{2}}
$$

and, in particular, (2.20) 3 .

Two interesting estimates have already been derived in [3. The first of these, which easily follows from (6.21), is

$$
D_{F}(t) \geq-\frac{1}{4} \int_{0}^{+\infty} \frac{d}{d \tau}\left[\frac{1}{\mu^{\prime}(\tau)}\right] \breve{\mathbf{I}}_{(1)}^{t}(\tau) \cdot \breve{\mathbf{I}}_{(1)}^{t}(\tau) d \tau \geq 0 .
$$

The other one is related to the particular case where there exists $\alpha \in \mathbb{R}^{++}$such that

$$
\mu^{\prime \prime}(\tau)+\alpha \mu^{\prime}(\tau) \geq 0 \quad \forall \tau \in \mathbb{R}^{+},
$$

so that we have

$$
-\mu^{\prime \prime}(\tau) \leq \alpha \mu^{\prime}(\tau), \quad-\frac{d}{d \tau}\left[\frac{1}{\mu^{\prime}(\tau)}\right] \geq-\frac{\alpha}{\mu^{\prime}(\tau)} .
$$

From the inequality (6.23) derived for $D_{F}$, by using the expression (6.13) for $\psi_{F}$, we also have

$$
D_{F}(t) \geq \alpha \psi_{F}(t),
$$

which gives an estimate of the internal dissipation in terms of just Fabrizio's free energy.

6.5. A general form for the free energy. We now consider the general representation of a free energy, the Breuer-Onat functiona 5 , which has already been studied for viscoelastic solids (see [4] and [5]) and, in particular, in the scalar case by Golden in [21]. Also this expression can be considered for fluids, and its form, already given in [3], is expressed as

$$
\psi(t)=\int_{0}^{+\infty} \int_{0}^{+\infty} \mu_{12}(s, u) \mathbf{E}_{r}^{t}(s) \cdot \mathbf{E}_{r}^{t}(u) d s d u
$$

where

$$
\mu_{12}(s, u)=\frac{\partial^{2}}{\partial s \partial u} \mu(s, u)
$$

must give non-negative values of the integral for all relative histories and be such that $\mu_{12}(+\infty, u)=\mu_{12}(s,+\infty)=0$, because the integral in (6.27) must exist for finite relative histories.

The time derivative of (6.27) has already been evaluated in [3]:

$$
\dot{\psi}(t)=\mathbf{T}(t) \cdot \dot{\mathbf{E}}(t)+\int_{0}^{+\infty} \int_{0}^{+\infty}\left[\mu_{1}(s, u)+\mu_{2}(s, u)\right] \dot{\mathbf{E}}^{t}(u) \cdot \dot{\mathbf{E}}^{t}(s) d s d u
$$

5 The most general quadratic form for the free energy is the Breuer-Onat functional, which corresponds to a linear non-aging material and a differentiable history. An alternative form, which does not require differentiability of the history of the strain, has been derived by Golden in the scalar case 21]. It can be written as

$$
\psi(t)=\frac{1}{2} \mathbf{E}(t) \cdot \mathbf{G}_{\infty} \mathbf{E}(t)+\frac{1}{2} \int_{0}^{+\infty} \int_{0}^{+\infty} \mathbf{E}_{r}^{t}(s) \cdot \mathbf{G}_{12}(s, u) \mathbf{E}_{r}^{t}(u) d s d u
$$

where the numerical subscripts on the fourth-order tensor $\mathbf{G}$ denote differentiation with respect to its arguments, that is, $\mathbf{G}_{12}(s, u)=\frac{\partial^{2}}{\partial s \partial u} \mathbf{G}(s, u)$, provided that $\mathbf{G}_{\infty}=\mathbf{G}(s,+\infty)=\mathbf{G}(+\infty, u)=\mathbf{G}(+\infty)$, $\mathbf{G}_{1}(s,+\infty)=\mathbf{G}_{2}(+\infty, u)=\mathbf{0}$ and $\mathbf{G}_{1}(s, 0)=\mathbf{G}_{2}(0, u)=\mathbf{G}^{\prime}(s)$, where $\mathbf{G}^{\prime}(s)$ is the derivative of the relaxation function $\mathbf{G}(s)$. 
on assuming that

$$
\mu_{1}(s,+\infty)=\mu_{2}(+\infty, u)=0, \quad \mu_{1}(s, 0)=\mu_{2}(0, s)=\mu^{\prime}(s) .
$$

The expression (6.29) satisfies (4.15) with an internal dissipation expressed by

$$
\begin{aligned}
D(t) & =-\int_{0}^{+\infty} \int_{0}^{+\infty} K(s, u) \dot{\mathbf{E}}^{t}(s) \cdot \dot{\mathbf{E}}^{t}(u) d s d u \\
& =-\int_{0}^{+\infty} \int_{0}^{+\infty} K_{12}(s, u) \mathbf{E}_{r}^{t}(s) \cdot \mathbf{E}_{r}^{t}(u) d s d u \geq 0,
\end{aligned}
$$

where we have integrated by parts and introduced the new kernel

$$
K(s, u)=\mu_{1}(s, u)+\mu_{2}(s, u), \quad K_{12}(s, u)=\frac{\partial^{2}}{\partial s \partial u} K(s, u),
$$

which must yield non-negative values of $D$ for all relative histories. We observe that the relation

$$
\mu(s, u)=\int_{s}^{+\infty} \int_{u}^{+\infty} \mu_{12}\left(s^{\prime}, u^{\prime}\right) d s^{\prime} d u^{\prime},
$$

satisfies (6.28) and is such that

$$
\mu(0, s)=\mu(s, 0)=\mu(s), \quad \mu(s,+\infty)=\mu(+\infty, u)=0 .
$$

Thus, after some integrations by parts, we can rewrite the expression (6.27) for $\psi(t)$ as

$$
\psi(t)=\int_{0}^{+\infty} \int_{0}^{+\infty} \mu(s, u) \dot{\mathbf{E}}^{t}(s) \cdot \dot{\mathbf{E}}^{t}(u) d s d u .
$$

REMARK 4. Keeping in mind the general form for the free energy expressed by (6.27), in [3] it has been possible to give the Fabrizio free energy $\psi_{F}$ another expression in terms of the relative strain history $\mathbf{E}_{r}^{t}$, to which $\breve{\mathbf{I}}_{(1)}^{t}(\tau)$ is related by virtue of (6.14), thus obtaining the following results. This new form is given by

$$
\begin{aligned}
\psi_{F}(t) & =-\int_{0}^{+\infty} \int_{0}^{+\infty} \int_{0}^{+\infty} \frac{1}{\mu^{\prime}(\tau)} \mu^{\prime \prime}\left(s_{1}+\tau\right) \mu^{\prime \prime}\left(s_{2}+\tau\right) \mathbf{E}_{r}^{t}\left(s_{1}\right) \cdot \mathbf{E}_{r}^{t}\left(s_{2}\right) d s_{1} d s_{2} d \tau \\
& =\int_{0}^{+\infty} \int_{0}^{+\infty} \mu_{12}\left(s_{1}, s_{2}\right) \mathbf{E}_{r}^{t}\left(s_{1}\right) \cdot \mathbf{E}_{r}^{t}\left(s_{2}\right) d s_{1} d s_{2},
\end{aligned}
$$

where we have introduced the function

$$
\mu\left(s_{1}, s_{2}\right)=-\int_{0}^{+\infty} \frac{1}{\mu^{\prime}(\tau)} \mu^{\prime}\left(s_{1}+\tau\right) \mu^{\prime}\left(s_{2}+\tau\right) d \tau=\mu\left(s_{2}, s_{1}\right),
$$

which satisfies (6.34) and yields, for the quantity $K$ in $(6.32) 1$, the following expression:

$$
\begin{aligned}
K\left(s_{1}, s_{2}\right) & =-\int_{0}^{+\infty} \frac{1}{\mu^{\prime}(\tau)}\left[\mu^{\prime \prime}\left(s_{1}+\tau\right) \mu^{\prime}\left(s_{2}+\tau\right)+\mu^{\prime}\left(s_{1}+\tau\right) \mu^{\prime \prime}\left(s_{2}+\tau\right)\right] d \tau \\
& \left.=\frac{1}{\mu^{\prime}(0)} \mu^{\prime}\left(s_{1}\right) \mu^{\prime}\left(s_{2}\right)+\int_{0}^{+\infty} \frac{d}{d \tau}\left[\frac{1}{\mu^{\prime}(\tau)}\right] \mu^{\prime}\left(s_{1}+\tau\right) \mu^{\prime}\left(s_{2}+\tau\right)\right] d \tau
\end{aligned}
$$

where we have carried out a partial integration with respect to $\tau$ and used (6.22). We see that $(\underline{6.36})_{1}$ yields a non-negative quantity for all relative histories by virtue of the hypotheses assumed on the derivatives of the kernel $\mu$. Moreover, the form $\left(\underline{6.36)_{2}} 2\right.$ is similar to the one in (6.27), to which corresponds the dissipation $D$ already derived in (6.31). 
Finally, by virtue of the assumptions on $\mu^{\prime}$ and $\mu^{\prime \prime}$, it follows that $K$, given by $(6.38)_{2}$, is non-positive; this result allows us to show again that the dissipation corresponding to Fabrizio's free energy is non-negative.

6.6. A special form for the general representation: the maximum free energy. A particular case of the general representation (6.27) is related to the assumption that $\mu_{12}(s, u)=$ $\mu_{12}(|s-u|)$; therefore, we consider the functiona 6

$$
\psi_{M}(t)=\int_{0}^{+\infty} \int_{0}^{+\infty} \mu_{12}(|s-u|) \mathbf{E}_{r}^{t}(s) \cdot \mathbf{E}_{r}^{t}(u) d s d u .
$$

Such a functional is particularly important because it yields the maximum free energy also for our fluids, just as it does for solids with the analogous functional.

To prove this property, we rewrite this expression by using the definition (2.2) of the relative strain history, as follows:

$$
\begin{aligned}
\psi_{M}(t) & =\int_{0}^{+\infty} \int_{0}^{+\infty} \mu_{12}(|s-u|) \mathbf{E}^{t}(s) \cdot \mathbf{E}^{t}(u) d s d u-\mathbf{E}(t) \\
& \cdot \int_{0}^{+\infty} \int_{0}^{+\infty} \mu_{12}(|s-u|)\left[\mathbf{E}^{t}(s)+\mathbf{E}^{t}(u)\right] d s d u+\mu_{0} \mathbf{E}(t) \cdot \mathbf{E}(t) \\
= & \mathbf{E}(t) \cdot\left[\mu_{0} \mathbf{E}(t)+2 \int_{0}^{+\infty} \mu^{\prime}(u) \mathbf{E}^{t}(u) d u\right] \\
& +\int_{0}^{+\infty} \int_{0}^{+\infty} \mu_{12}(|s-u|) \mathbf{E}^{t}(s) \cdot \mathbf{E}^{t}(u) d s d u,
\end{aligned}
$$

because

$$
\int_{0}^{+\infty} \int_{0}^{+\infty} \mu_{12}(|s-u|) d s d u=\mu_{0}
$$

We observe that, in particular, from (6.40) we can deduce the property of a free energy expressed by $\partial \psi_{M} / \partial \mathbf{E}=\tilde{\mathbf{T}}$. Then, the derivative with respect to $t$ yields

$$
\begin{aligned}
\dot{\psi}_{M}(t)= & {\left[\mu_{0} \mathbf{E}(t)+2 \int_{0}^{+\infty} \mu^{\prime}(u) \mathbf{E}^{t}(u) d u\right] \cdot \dot{\mathbf{E}}(t)+2 \mathbf{E}(t) \cdot \int_{0}^{+\infty} \mu^{\prime}(u) \dot{\mathbf{E}}^{t}(u) d u } \\
& +2 \int_{0}^{+\infty} \int_{0}^{+\infty} \mu_{12}(|s-u|) \dot{\mathbf{E}}^{t}(s) \cdot \mathbf{E}^{t}(u) d s d u
\end{aligned}
$$

Now, we note that the last integral of (6.42), after an integration by parts, gives

$$
\begin{aligned}
& 2 \int_{0}^{+\infty} \int_{0}^{+\infty} \mu_{12}(|s-u|) \dot{\mathbf{E}}^{t}(s) \cdot \mathbf{E}^{t}(u) d s d u \\
& =2 \int_{0}^{+\infty} \int_{0}^{+\infty} \mu_{1}(|s-u|) \dot{\mathbf{E}}^{t}(s) \cdot \dot{\mathbf{E}}^{t}(u) d s d u-2 \int_{0}^{+\infty} \mu_{1}(s) \dot{\mathbf{E}}^{t}(s) d s \cdot \mathbf{E}(t),
\end{aligned}
$$

\footnotetext{
${ }^{6}$ A particular case of the expression for the general form of the free energy, expressed in terms of the functional of the relative history $\mathbf{E}_{r}^{t}$ with the kernel $\mathbf{G}_{12}(s, u)$ considered in the previous note, is obtained by supposing that $\mathbf{G}_{12}\left(s_{1}, s_{2}\right)=\mathbf{G}_{12}\left(\left|s_{1}-s_{2}\right|\right)$. The corresponding functional, considered in [16], has the form

$$
\psi_{M}\left(\mathbf{E}^{t}\right)=\frac{1}{2} \mathbf{E}(t) \cdot \mathbf{G}_{\infty} \mathbf{E}(t)+\frac{1}{2} \int_{0}^{+\infty} \int_{0}^{+\infty} \mathbf{E}_{r}^{t}\left(s_{1}\right) \cdot \mathbf{G}_{12}\left(\left|s_{1}-s_{2}\right|\right) \mathbf{E}_{r}^{t}\left(s_{2}\right) d s_{1} d s_{2},
$$

where $\mathbf{G}_{12}=\frac{\partial^{2} \mathbf{G}\left(\left|s_{1}-s_{2}\right|\right)}{\partial s_{1} \partial s_{2}}$. It is very interesting since it expresses the maximum free energy for viscoelastic solids.
} 
the first term of which is equal to zero because of the oddness of

$$
\frac{\partial \mu}{\partial s}(|s-u|)=\operatorname{sign}(s-u) \mu^{\prime}(|s-u|),
$$

and the second term of which cancels the third term in (6.42). Consequently, using the constitutive equation (2.1) and the definition (2.2) of $\mathbf{E}_{r}^{t}$, (6.42) reduces to

$$
\dot{\psi}_{M}(t)=\mathbf{T}(t) \cdot \dot{\mathbf{E}}(t),
$$

by virtue of the constraint of incompressibility (5.1). Hence, it follows that (4.15) is satisfied without dissipation, that is,

$$
D_{M}(t)=0 .
$$

We can show the following lemma easily.

Lemma 5. The functional $\psi_{M}$ is the maximum free energy.

Proof. Let $P$ be an arbitrary process, applied at time $t=0$ to the zero state $\sigma^{\dagger}=$ $\mathbf{E}_{r}^{0}(s)=\mathbf{0}_{r}^{\dagger}(s)=\mathbf{0} \forall s \in \mathbb{R}^{++}$. We denote by $\sigma(t)=\mathbf{E}_{r}^{t}=\rho\left(\mathbf{0}_{r}^{\dagger}, P_{[0, t)}\right)$ the final state at time $t>0$; this state belongs to

$$
\mathcal{D}_{\psi_{M}}=\left\{\mathbf{E}_{r}^{t} ; \psi_{M}\left(\mathbf{E}_{r}^{t}\right)<+\infty\right\},
$$

the set of all relative histories which yield a finite value for $\psi_{M}(\sigma(t))=\psi_{M}\left(\mathbf{E}_{r}^{t}\right)$.

By integrating (6.44) over the time interval $(0, t)$, we obtain

$$
\psi_{M}(\sigma(t))=\int_{0}^{t} \mathbf{T}(s) \cdot \dot{\mathbf{E}}(s) d s,
$$

since $\psi_{M}\left(\sigma^{\dagger}\right)=0$. Moreover, let $\psi(\sigma(t))$, where $\sigma(t)=\hat{\rho}\left(\mathbf{0}_{r}^{\dagger}, P_{[0, t)}\right)$, be any other free energy. Then, we have the inequality

$$
\psi(\sigma(t)) \leq \int_{0}^{t} \mathbf{T}(s) \cdot \dot{\mathbf{E}}(s) d s,
$$

because of (4.7) and the fact that $\psi\left(\sigma^{\dagger}\right)=0$. From (6.47) and (6.48) we get the inequality

$$
\psi_{M}(\sigma) \geq \psi(\sigma),
$$

where $\sigma(t)=\mathbf{E}_{r}^{t} \in \mathcal{D}_{\psi_{M}}$ is an arbitrary final state, since it is obtained by means of any process $P$. This inequality holds whatever the functional $\psi$ assumed as free energy may be; consequently, $\psi_{M}$ is the maximum free energy for viscoelastic fluids.

6.7. Golden's free energy: the minimum free energy. It is well known that the minimum free energy coincides with the maximum recoverable work that we can obtain from a given state $\sigma$ of the material. This quantity is given by (4.9) or equivalently by

$$
\psi_{m}(\sigma)=W_{R}(\sigma)=\sup \{-W(\sigma, P): P \in \Pi\},
$$

where $\Pi$ is the set of finite work processes. 
The expression for the minimum free energy, related to linear viscoelastic solids, was derived in the scalar case by Golden 7 in 21 .

As has been done for viscoelastic solids (see [21] and 20]), two equivalent expressions for the minimum free energy have been derived also for viscoelastic fluids (see [1, 2] and (3)).

The first of these formulations was deduced by considering a function $\mathcal{K}$, with no zeros at any $\omega \in \mathbb{R}$ or at infinity, such that

$$
\mathcal{K}(\omega)=\left(1+\omega^{2}\right) \mu_{c}(\omega), \quad \mathcal{K}(\omega)=\mathcal{K}_{(+)}(\omega) \mathcal{K}_{(-)}(\omega),
$$

from which a factorization of $\mu_{c}(\omega)$ can be derived in the form

$$
\mu_{c}(\omega)=\mu_{(+)}(\omega) \mu_{(-)}(\omega), \quad \mu_{( \pm)}(\omega)=\frac{\mathcal{K}_{( \pm)}(\omega)}{1 \pm i \omega},
$$

where the subscript ${ }_{( \pm)}$is used to indicate that the corresponding function of $\omega$, considered as a function of $z \in \mathbb{C}$, has zeros and singularities only for $z \in \mathbb{C}^{ \pm}$.

Then, using the Plemelj formulae [28], which give

$$
\frac{1}{4 \mu_{(-)}(\omega)} \breve{\mathbf{I}}_{+}^{t}(\omega)=\mathbf{p}_{(-)}^{t}(\omega)-\mathbf{p}_{(+)}^{t}(\omega)
$$

with

$$
\mathbf{p}^{t}(z)=\frac{1}{2 \pi i} \int_{-\infty}^{+\infty} \frac{\breve{\mathbf{I}}_{+}^{t}(\omega) / 4 \mu_{(-)}(\omega)}{\omega-z} d \omega, \quad \mathbf{p}_{( \pm)}^{t}(\omega)=\lim _{\beta \rightarrow 0^{\mp}} \mathbf{p}^{t}(\omega+i \beta),
$$

where $\mathbf{p}_{( \pm)}^{t}$ is analytic in $\mathbb{C}^{(\mp)}$ and also in $\mathbb{R}$ by the hypothesis on the Fourier transforms, we obtain the expression for the Golden free energy for fluids, that is,

$$
\psi_{\text {Golden }}(t) \equiv \psi_{m}(t)=\frac{1}{\pi} \int_{-\infty}^{+\infty}\left|\mathbf{p}_{(+)}^{t}(\omega)\right|^{2} d \omega
$$

7 Another important functional which gives a free energy for viscoelastic solids is the Golden free energy [21]. Its importance is due to the fact that it gives the minimum free energy for these materials. For such a functional two equivalent representations have been derived in the frequency domain (see 10] and [11]), one of which is expressed in terms of the Fourier transform of the relative history $\mathbf{E}_{r+}^{t}$, and the other by means of the Fourier transform of the past history $\mathbf{E}_{+}^{t}$. We recall only the first of these expressions, which can be written as

$$
\psi_{\text {Golden }}(t) \equiv \psi_{m}(t)=\frac{1}{2} \mathbf{E}(t) \cdot \mathbf{G}_{\infty} \mathbf{E}(t)+\frac{1}{2 \pi} \int_{-\infty}^{+\infty}\left|\mathbf{p}_{-}^{t}(\omega)\right|^{2} d \omega .
$$

The particular notation used in this expression is related to the quantity

$$
\mathbf{p}_{-}^{t}(\omega)=\lim _{z \rightarrow \omega^{+}} \mathbf{p}^{t}(z) \equiv \lim _{z \rightarrow \omega^{+}} \frac{1}{2 \pi i} \int_{-\infty}^{+\infty} \frac{\mathbf{H}_{-}\left(\omega^{\prime}\right) \mathbf{E}_{r+}^{t}\left(\omega^{\prime}\right)}{\omega^{\prime}-z} d \omega^{\prime}, \quad \omega^{+}=\lim _{\alpha \rightarrow 0^{+}}(\omega+i \alpha),
$$

which admits an analytic extension $\mathbf{p}^{t}(z)=\mathbf{p}_{-}^{t}(z)$ for any $z \in \mathbb{C}^{(+)}$, and also on $\mathbb{R}$ by virtue of the assumption on the analyticity of any Fourier transform. Moreover, the sign used, in particular, in $\mathbf{H}_{-}(\omega)$, obtained by means of the factorization of the fourth-order tensor $\mathbf{H}(\omega) \equiv-\omega \mathbf{G}_{s}^{\prime}(\omega)=\mathbf{H}_{+}(\omega) \mathbf{H}_{-}(\omega)$, indicates the half plane where any singularities of the tensor and any zeros in the determinant of the corresponding matrix occur. Finally, $\mathbf{G}_{s}^{\prime}(\omega)$ is the half-range Fourier sine transform of the relaxation function $\mathbf{G}$. 
The second expression for $\psi_{m}$ was derived by evaluating a new expression for $\mathbf{p}_{(+)}^{t}(\omega)$ as a function of $\mathbf{E}_{r+}^{t}(\omega)$, that is,

$$
\mathbf{p}_{(+)}^{t}(\omega)=-\lim _{z \rightarrow \omega^{-}} \frac{1}{2 \pi i} \int_{-\infty}^{+\infty} \frac{i \omega^{\prime} \mu_{(+)}\left(\omega^{\prime}\right)\left[\mathbf{E}_{r+}^{t}\left(\omega^{\prime}\right)\right]^{*}}{\omega^{\prime}-z} d \omega^{\prime},
$$

where

$$
\omega^{ \pm}=\lim _{\alpha \rightarrow 0^{+}}(\omega \pm i \alpha)
$$

Thus, putting

$$
\left[\mathbf{p}_{(+)}^{t}(\omega)\right]^{*}=-\lim _{\xi \rightarrow \omega+} \frac{1}{2 \pi i} \int_{-\infty}^{+\infty} \frac{i \omega^{\prime} \mu_{(-)}\left(\omega^{\prime}\right) \mathbf{E}_{r+}^{t}\left(\omega^{\prime}\right)}{\omega^{\prime}-\xi} d \omega^{\prime}=i \tilde{\mathbf{q}}_{(-)}^{t}(\omega),
$$

that is,

$$
\tilde{\mathbf{q}}_{( \pm)}^{t}(\omega)=\lim _{\xi \rightarrow \omega \mp} \frac{1}{2 \pi i} \int_{-\infty}^{+\infty} \frac{-\omega^{\prime} \mu_{(-)}\left(\omega^{\prime}\right) \mathbf{E}_{r+}^{t}\left(\omega^{\prime}\right)}{\omega^{\prime}-\xi} d \omega^{\prime},
$$

we consider the relation

$$
\tilde{\mathbf{q}}_{(-)}^{t}(\omega)-\tilde{\mathbf{q}}_{(+)}^{t}(\omega)=-\omega \mu_{(-)}(\omega) \mathbf{E}_{r+}^{t}(\omega),
$$

thus obtaining the other equivalent expression for the Golden free energy:

$$
\psi_{\text {Golden }}(t) \equiv \psi_{m}(t)=\frac{1}{\pi} \int_{-\infty}^{+\infty}\left|\tilde{\mathbf{q}}_{(-)}^{t}(\omega)\right|^{2} d \omega,
$$

which expresses the other form of the minimum free energy for our fluids.

We note that in the first representation (6.55) the quantity $\breve{\mathbf{I}}^{t}$, which is the minimal state also for our fluids, is involved, while in the second expression (6.61) $\psi_{m}$ is given by means of the relative history of the strain $\mathbf{E}_{r}^{t}$.

7. The minimum free energy for a discrete spectrum model and its properties. An interesting model, already considered for linear viscoelastic solids in the scalar case by Golden in [21, is characterized by a relaxation function given by a sum of exponentials, namely the discrete spectrum model. Already in 21], Golden derived the corresponding minimum free energy with related internal dissipation, by using the frequency domain; moreover, a comparison of these results with some free energies has been undertaken.

Also for our fluids, this method has been applied in 2]; the results obtained there are now reviewed in order to compare them with known free energies.

Let the relaxation function $\mu$ have the form

$$
\mu(t)= \begin{cases}\sum_{i=1}^{n} \mu_{i} e^{-\alpha_{i} t} & \forall t \geq 0, \\ 0 & \forall t<0,\end{cases}
$$

where $n$ is a positive integer and $\alpha_{i}$ and $\mu_{i}(i=1,2, \ldots, n)$ are positive coefficients. We observe that (2.18) is satisfied since $\mu(0)=\sum_{i=1}^{n} \mu_{i}>0$; moreover, $\alpha_{i}(i=1,2, \ldots, n)$ can 
be ordered so that $\alpha_{1}<\alpha_{2}<\ldots<\alpha_{n}$. The Fourier transform of $\mu$, upon using (6.51), yields

$$
\mu_{c}(\omega)=\sum_{i=1}^{n} \frac{\alpha_{i} \mu_{i}}{\alpha_{i}^{2}+\omega^{2}}, \quad \mathcal{K}(\omega)=\sum_{i=1}^{n} \alpha_{i} \mu_{i} \frac{1+\omega^{2}}{\alpha_{i}^{2}+\omega^{2}} .
$$

The function $\mathcal{K}(\omega)$ can be rewritten as

$$
\mathcal{K}(\omega)=\mathcal{K}_{\infty} \prod_{i=1}^{n}\left\{\frac{\gamma_{i}^{2}+\omega^{2}}{\alpha_{i}^{2}+\omega^{2}}\right\}, \quad \mathcal{K}_{\infty}=\lim _{\omega \rightarrow \infty} \mathcal{K}(\omega)=\sum_{i=1}^{n} \alpha_{i} \mu_{i}>0,
$$

where $\gamma_{1}^{2}=1$ and $\gamma_{j}^{2}(j=2,3, \ldots, n)$ denote the zeros of $f(z)=\mathcal{K}(\omega)$ with $z=-\omega^{2}$; these are simple, or at most only one of the values $\gamma_{j}^{2}$ can coincide with $\gamma_{1}^{2}=1$, which then becomes a zero of multiplicity 2 . The factorization of $(\mathbf{7 . 3})$, by virtue of $(6.52){ }_{2}$, yields

$$
\omega \mu_{(-)}(\omega)=i k_{\infty} \prod_{i=1}^{n}\left\{\frac{\omega+i \delta_{i}}{\omega+i \alpha_{i}}\right\}=i k_{\infty}\left(1+i \sum_{r=1}^{n} \frac{A_{r}}{\omega+i \alpha_{r}}\right), k_{\infty}=\left(\mathcal{K}_{\infty}\right)^{1 / 2},
$$

with

$$
A_{r}=\left(\delta_{r}-\alpha_{r}\right) \prod_{i=1, i \neq r}^{n}\left\{\frac{\delta_{i}-\alpha_{r}}{\alpha_{i}-\alpha_{r}}\right\} \quad(r=1,2, \ldots, n),
$$

where $\delta_{1}=\gamma_{0}=0$ and $\delta_{j}=\gamma_{j}(j=2,3, \ldots, n)$.

Thus, by using (7.4), the quantity $\tilde{\mathbf{q}}_{(-)}^{t}(\omega)$, defined in (6.59), may be obtained by closing the contour on $\mathbf{C}^{(-)}$, giving

$$
\tilde{\mathbf{q}}_{(-)}^{t}(\omega)=k_{\infty} \sum_{i=1}^{n} A_{i} \frac{\mathbf{E}_{r+}^{t}\left(-i \alpha_{i}\right)}{\omega+i \alpha_{i}},
$$

where, by virtue of $(\underline{2.6})_{2}$,

$$
\mathbf{E}_{r+}^{t}\left(-i \alpha_{i}\right)=\int_{0}^{+\infty} \mathbf{E}_{r}^{t}(s) e^{-\alpha_{i} s} d s=\left[\mathbf{E}_{r+}^{t}\left(-i \alpha_{i}\right)\right]^{*} .
$$

The complex conjugate of (17.6) allows us to derive the term $\left|\tilde{\mathbf{q}}_{(-)}^{t}(\omega)\right|^{2}$ present in (6.61), which, by closing the contour now in $\mathbf{C}^{(+)}$, yields

$$
\psi_{m}(t)=2 \mathcal{K}_{\infty} \sum_{i, j=1}^{n} \frac{A_{i} A_{j}}{\alpha_{i}+\alpha_{j}} \int_{0}^{+\infty} \int_{0}^{+\infty} e^{-\left(\alpha_{i} s_{1}+\alpha_{j} s_{2}\right)} \mathbf{E}_{r}^{t}\left(s_{1}\right) \cdot \mathbf{E}_{r}^{t}\left(s_{2}\right) d s_{1} d s_{2} .
$$

Also for our fluids it is possible to compare (7.8) with other free energies.

In fact, we can rewrite (7.8) in the form

$$
\psi_{m}(t)=\int_{0}^{+\infty} \int_{0}^{+\infty} \Gamma\left(s_{1}, s_{2}\right) \mathbf{E}_{r}^{t}\left(s_{1}\right) \cdot \mathbf{E}_{r}^{t}\left(s_{2}\right) d s_{1} d s_{2},
$$

by introducing the kernel $\Gamma\left(s_{1}, s_{2}\right)$ deduced from (17.8) and given by

$$
\Gamma\left(s_{1}, s_{2}\right) \equiv \mu_{12}\left(s_{1}, s_{2}\right)=2 \mathcal{K}_{\infty} \sum_{i, j=1}^{n} \frac{A_{i} A_{j}}{\alpha_{i}+\alpha_{j}} e^{-\left(\alpha_{i} s_{1}+\alpha_{j} s_{2}\right)} ;
$$

therefore, the expression for the minimum free energy (17.8) is cast in the general form for the free energy introduced in (6.27). 
We can now solve the differential equation $(17.10)_{2}$ in order to obtain the solution $\mu\left(s_{1}, s_{2}\right)$. Taking account of (6.33), we have

$$
\mu\left(s_{1}, s_{2}\right)=2 \mathcal{K}_{\infty} \sum_{i, j=1}^{n} \frac{1}{\alpha_{i}+\alpha_{j}} \frac{A_{i}}{\alpha_{i}} \frac{A_{j}}{\alpha_{j}} e^{-\left(\alpha_{i} s_{1}+\alpha_{j} s_{2}\right)} .
$$

This solution must satisfy the assumptions (6.30) and (6.34). To verify this, we need some useful identities, which are similar to those already derived by Golden in [21]. For our fluids they become

$$
\begin{aligned}
& \sum_{i=1}^{n} \frac{A_{i}}{\alpha_{i}}=-1, \quad \sum_{j=1}^{n} \frac{A_{j}}{\left(\alpha_{i}+\alpha_{j}\right) \alpha_{j}}=\frac{\mu_{i} \alpha_{i}}{2 \mathcal{K}_{\infty} A_{i}}, \\
& \sum_{j=1}^{n} \frac{A_{j}}{\alpha_{i}+\alpha_{j}}=-1-\frac{\mu_{i} \alpha_{i}^{2}}{2 \mathcal{K}_{\infty} A_{i}}, \quad A_{i} B_{i}=-\frac{\mu_{i} \alpha_{i}^{3}}{\mathcal{K}_{\infty}}
\end{aligned}
$$

where

$$
B_{i}=\left(\delta_{i}+\alpha_{i}\right) \prod_{j=1, j \neq i}^{n}\left\{\frac{\delta_{j}+\alpha_{i}}{\alpha_{j}+\alpha_{i}}\right\}>0 \quad(i=1,2, \ldots, n) .
$$

These easily follow, respectively, from (7.4) ${ }_{1,2}$ for $\omega=0$, from (7.11) and (7.1) upon comparing the product of $\omega \mu_{(-)}(\omega)$, given by $\left({ }^{7.4}\right)_{2}$, and its conjugate with $\omega^{2} \mu_{c}(\omega)$, given by (7.2) $)_{1}$, near the poles of $\omega \mu_{(+)}(\omega)$, and from comparing the two expressions for $\omega^{2} \mu_{c}(\omega)$ derived via multiplying $(17.2)_{1}$ by $\omega^{2}$ and $(6.51)_{1}$ by $\frac{\omega^{2}}{1+\omega^{2}}$, using the expression $(7.3)_{1}$ in terms of $\delta_{i}(i=1,2, \ldots, n)$.

Thus, the function (7.11) satisfies (6.34) as well as (6.30) by virtue of the identity $(7.12)_{2}$.

We can rewrite (7.11) by substituting the expression for $A_{i}$ given by $(\mathbf{7 . 1 3})_{2}$, as follows:

$$
\mu\left(s_{1}, s_{2}\right)=\frac{2}{\mathcal{K}_{\infty}} \sum_{i, j=1}^{n} \frac{\alpha_{i}^{2} \alpha_{j}^{2} \mu_{i} \mu_{j}}{\left(\alpha_{i}+\alpha_{j}\right) B_{i} B_{j}} e^{-\left(\alpha_{i} s_{1}+\alpha_{j} s_{2}\right)}>0,
$$

which yields the equivalence to the Breuer-Onat formula and, therefore, to the alternative form proposed by Golden, that is, (6.27), which, on account of (7.9), (7.10) and (7.13) 2 , becomes

$$
\psi_{m}(t)=\frac{2}{\mathcal{K}_{\infty}} \int_{0}^{+\infty} \int_{0}^{+\infty} \sum_{i, j=1}^{n} \frac{\alpha_{i}^{3} \alpha_{j}^{3} \mu_{i} \mu_{j}}{\left(\alpha_{i}+\alpha_{j}\right) B_{i} B_{j}} e^{-\left(\alpha_{i} s_{1}+\alpha_{j} s_{2}\right)} \mathbf{E}_{r}^{t}\left(s_{1}\right) \cdot \mathbf{E}_{r}^{t}\left(s_{2}\right) d s_{1} d s_{2} .
$$

From (6.32) we can also derive the form of $K\left(s_{1}, s_{2}\right)$ which appears in the expression for the rate of dissipation (6.31) 1 , as well as the one for $K_{12}\left(s_{1}, s_{2}\right)$ present in (6.31) 2 . In fact, using (6.32) 1 and (7.11), we have

$$
K\left(s_{1}, s_{2}\right)=-2 \mathcal{K}_{\infty} \sum_{i, j=1}^{n} \frac{A_{i}}{\alpha_{i}} \frac{A_{j}}{\alpha_{j}} e^{-\left(\alpha_{i} s_{1}+\alpha_{j} s_{2}\right)}
$$


and hence

$$
\begin{aligned}
K_{12}\left(s_{1}, s_{2}\right) & =-2 \mathcal{K}_{\infty} \sum_{i, j=1}^{n} A_{i} A_{j} e^{-\left(\alpha_{i} s_{1}+\alpha_{j} s_{2}\right)} \\
& =-\frac{2}{\mathcal{K}_{\infty}} \sum_{i, j=1}^{n} \frac{\alpha_{i}^{3} \alpha_{j}^{3} \mu_{i} \mu_{j}}{B_{i} B_{j}} e^{-\left(\alpha_{i} s_{1}+\alpha_{j} s_{2}\right)}<0 .
\end{aligned}
$$

Therefore, we obtain the corresponding internal dissipation $(6.31)_{2}$, i.e.

$$
D_{m}(t)=\frac{2}{\mathcal{K}_{\infty}} \int_{0}^{+\infty} \int_{0}^{+\infty} \sum_{i, j=1}^{n} \frac{\alpha_{i}^{3} \alpha_{j}^{3} \mu_{i} \mu_{j}}{B_{i} B_{j}} e^{-\left(\alpha_{i} s_{1}+\alpha_{j} s_{2}\right)} \mathbf{E}_{r}^{t}\left(s_{1}\right) \cdot \mathbf{E}_{r}^{t}\left(s_{2}\right) d s_{1} d s_{2}
$$

where all the coefficients are positive, by virtue of $(\overline{(7.3)})_{2}$ and (7.14) in particular. This quantity can be expressed in a form which is clearly non-negative, because it can be written as

$$
\begin{aligned}
D_{m}(t)= & \frac{2}{\mathcal{K}_{\infty}}\left(\sum_{i=1}^{n} \frac{\alpha_{i}^{3} \mu_{i}}{B_{i}} \int_{0}^{+\infty} e^{-\alpha_{i} s_{1}} \mathbf{E}_{r}^{t}\left(s_{1}\right) d s_{1}\right) \\
& \cdot\left(\sum_{j=1}^{n} \frac{\alpha_{j}^{3} \mu_{j}}{B_{j}} \int_{0}^{+\infty} e^{-\alpha_{j} s_{2}} \mathbf{E}_{r}^{t}\left(s_{2}\right) d s_{2}\right) \\
= & \frac{2}{\mathcal{K}_{\infty}}\left(\sum_{i=1}^{n} \frac{\alpha_{i}^{3} \mu_{i}}{B_{i}} \int_{0}^{+\infty} e^{-\alpha_{i} s} \mathbf{E}_{r}^{t}(s) d s\right)^{2} \geq 0 .
\end{aligned}
$$

In the particular case where $n=1,(7.4)$ becomes

$$
\omega \mu_{(-)}(\omega)=i k_{\infty} \frac{\omega}{\omega+i \alpha_{1}}=i k_{\infty}\left(1+i \frac{A_{1}}{\omega+i \alpha_{1}}\right), A_{1}=-\alpha_{1}, \mathcal{K}_{\infty}=\alpha_{1} \mu_{1} .
$$

Hence, (6.59) reduces to

$$
\tilde{\mathbf{q}}_{(-)}^{t}(\omega)=k_{\infty} A_{1} \frac{\mathbf{E}_{r+}^{t}\left(-i \alpha_{1}\right)}{\omega+i \alpha_{1}},
$$

which gives the $\left|\tilde{\mathbf{q}}_{(-)}^{t}(\omega)\right|^{2}$ present in (6.61). Thus, by closing the contour again on $\mathbf{C}^{(+)}$, we obtain

$$
\psi_{m}(t)=\mu_{1} \alpha_{1}^{2}\left[\int_{0}^{+\infty} e^{-\alpha_{1} s} \mathbf{E}_{r}^{t}(s) d s\right]^{2},
$$

which agrees with the Day functional (6.4), as can be shown by putting in (6.4)

$$
\mu(s)=\mu_{1} e^{-\alpha_{1} s}, \quad \gamma=\alpha_{1} .
$$

Moreover, (6.9) and (17.24) yield the expression for the corresponding internal dissipation:

$$
D_{m}(t)=\frac{2 \alpha_{1}}{\mu_{1}}\left[-\int_{0}^{+\infty} \alpha_{1} \mu_{1} e^{-\alpha_{1} s} \mathbf{E}_{r}^{t}(s) d s\right]^{2}=2 \alpha_{1}^{3} \mu_{1}\left[\int_{0}^{+\infty} e^{-\alpha_{1} s} \mathbf{E}_{r}^{t}(s) d s\right]^{2} .
$$




\section{REFERENCES}

[1] G. Amendola, The minimum free energy for incompressible viscoelastic fluids, Math. Meth. Appl. Sci. 29, 2201-2223 (2006). MR2273157 (2008a:76010)

[2] G. Amendola and M. Fabrizio, Maximum recoverable work for incompressible viscoelastic fluids and application to a discrete spectrum model, Diff. Int. Eq. 20 (4), 445-466 (2007). MR2307142 (2008c:74018)

[3] G. Amendola, M. Fabrizio and J.M. Golden, Maximum recoverable work and free energies for incompressible viscoelastic fluids, Ukrainian Mathematical Journal, to appear.

[4] S. Breuer and E.T. Onat, On recoverable work in linear viscoelasticity, Z. Angew. Math. Phys. 15, 12-21 (1964). MR0178644 (31:2901)

[5] S. Breuer and E.T. Onat, On the determination of free energy in linear viscoelastic solids, Z. Angew. Math. Phys. 15, 184-191 (1964). MR0178645(31:2902)

[6] B.D. Coleman and W. Noll, Foundation of linear viscoelasticity, Rev. Modern Phys. 33, 239-249 (1961). MR0158605 (28:1828)

[7] B.D. Coleman and R.D. Owen, A mathematical foundation for thermodynamics, Arch. Rational Mech. Anal. 54, 1-104 (1974). MR0395502 (52:16299)

[8] W.A. Day, Reversibility, recoverable work and free energy in linear viscoelasticity, Quart. J. Mech. Appl. Math. 23, 1-14 (1970). MR0273881 (42:8757)

[9] G. Del Piero and L. Deseri, On the analytic expression of the free energy in linear viscoelasticity, J. Elasticity 43, 247-278 (1996). MR:1415545 (97g:73042)

[10] L. Deseri, M. Fabrizio and J.M. Golden, The concept of a minimal state in viscoelasticity: new free energies and applications to PDEs, Arch. Rational Mech. Anal. 181 (1), 43-96 (2006). MR2221203 (2009a:74024)

[11] L. Deseri, G. Gentili and J.M. Golden, An explicit formula for the minimum free energy in linear viscoelasticity, J. Elasticity 54, 141-185 (1999). MR.1728444(2001i:74012)

[12] E.D. Dill, Simple materials with fading memory, in: Continuum Physics II, Academic Press, Berlin, 1972 .

[13] M. Fabrizio, Free energies in the materials with fading memory and applications to PDEs, in: Proceedings WASCOM 2003, World Scientific, Singapore, 2004. MR2089846

[14] M. Fabrizio and C. Giorgi, Sulla termodinamica dei materiali semplici, Boll. Un. Mat. Ital. (6) 5-B, 441-464 (1986).

[15] M. Fabrizio, C. Giorgi and A. Morro, Free energies and dissipation properties for systems with memory, Arch. Rational Mech. Anal. 125, 341-373 (1994). MR.1253168 (95j:73012)

[16] M. Fabrizio, C. Giorgi and A. Morro, Internal dissipation, relaxation property and free energy in materials with fading memory, J. Elasticity 40, 107-122 (1995). MR1364749 (96g:73008)

[17] M. Fabrizio and J.M. Golden, Maximum and minimum free energies for a linear viscoelastic material, Quart. Appl. Math. LX (2), 341-381 (2002). MR.1900497(2003b:74013)

[18] M. Fabrizio and B. Lazzari, On asymptotic stability for linear viscoelastic fluids, Diff. Int. Eq. 6 (3), 491-504 (1993). MR1202554 (94b:76009)

[19] M. Fabrizio and A. Morro, Mathematical Problems in Linear Viscoelasticity, SIAM, Philadelphia, 1992. MR1153021 (93a:73034)

[20] G. Gentili, Maximum recoverable work, minimum free energy and state space in linear viscoelasticity, Quart. Appl. Math. LX (1), 153-182 (2002). MR1878264 (2002m:74011)

[21] J.M. Golden, Free energies in the frequency domain: the scalar case, Quart. Appl. Math. 58, 127-150 (2000). MR1739041 (2001e:74025)

[22] D. Graffi, Sull'espressione analitica di alcune grandezze termodinamiche nei materiali con memoria, Rend. Sem. Mat. Univ. Padova 68, 17-29 (1982).

[23] D. Graffi, Ancora sull'espressione dell'energia libera nei materiali con memoria, Atti Acc. Scienze Torino 120, 111-124 (1986). MR958166 (90f:73030)

[24] D. Graffi and M. Fabrizio, Non unicità dell'energia libera per materiali viscoelastici, Atti Accad. Naz. Lincei 83, 209-214 (1990). MR1142460 (93a:73037)

[25] D.D. Joseph, Slow motion and viscometric motion; stability and bifurcation of the rest state of a simple fluid, Arch. Rational Mech. Anal. 56, 99-157 (1974). MR0351255 (50:3744)

[26] D.D. Joseph, Fluid Dynamics of Viscoelastic Liquids , Springer Verlag, New York, 1990. MR.1051193 (91d:76003) 
[27] A. Morro and M. Vianello, Minimal and maximal free energy for materials with fading memory, Boll. Un. Mat. Ital. A 4, 45-55 (1990). MR.1047512 (91b:73012)

[28] N.I. Muskhelishvili, Singular Integral Equations, Noordhoff, Groningen, 1953. MR0355494(50:7968)

[29] M. Slemrod, A hereditary partial differential equation with applications in the theory of simple fluids, Arch. Rational Mech. Anal. 62, 303-332 (1976). MR0416245(54:4320)

[30] M. Slemrod, An energy stability method for simple fluids, Arch. Rational Mech. Anal. 68, 1-18 (1978). MR0503004 (58:19873)

[31] C. Truesdell, The meaning of viscometry in fluid dynamics, Ann. Rev. Fluid Mech. 6, 111-146 (1974).

[32] V. Volterra, Theory of Functional and of Integral and Integro-Differential Equations, Blackie \& Son Limited, London, 1930. 